\title{
Determination of the event collision time with the ALICE detector at the LHC
}

\author{
ALICE Collaboration ${ }^{\mathrm{a}, \mathrm{b}}$ \\ European Organization for Nuclear Research (CERN), Geneva, Switzerland
}

Received: 20 October 2016

Published online: 24 February 2017

(C) CERN for the benefit of the ALICE Collaboration 2017. This article is published with open access at Springerlink.com

\begin{abstract}
Particle identification is an important feature of the ALICE detector at the LHC. In particular, for particle identification via the time-of-flight technique, the precise determination of the event collision time represents an important ingredient of the quality of the measurement. In this paper, the different methods used for such a measurement in ALICE by means of the T0 and the TOF detectors are reviewed. Efficiencies, resolution and the improvement of the particle identification separation power of the methods used are presented for the different LHC colliding systems (pp, p-Pb and $\mathrm{Pb}-\mathrm{Pb}$ ) during the first period of data taking of LHC (RUN 1).
\end{abstract}

\section{Introduction}

The main task of the ALICE experiment $[1,2]$ at the LHC is the study of the properties of the strongly interacting, dense and hot matter created in high-energy heavy-ion collisions. Many physics analyses are based on the capability of the ALICE detector to perform Particle IDentification (PID) using different and complementary techniques. In the intermediate momentum range (from 0.5 to $3-4 \mathrm{GeV} / c$ ) this task is mainly accomplished using the time-of-flight measurements which rely on a precise determination of the event collision time, the track length and momentum, and the arrival time of the tracks to the Time-of-flight (TOF) detector.

The track length and momentum measurement is defined by the Inner Tracking System (ITS) and the Time Projection Chamber (TPC) [3]. The ITS is composed of six cylindrical layers of silicon detectors, located at radial distances between 3.9 and $43 \mathrm{~cm}$ from the beam axis. The TPC is a large volume cylindrical chamber with highgranularity readout that surrounds the ITS covering the region $85<r<247 \mathrm{~cm}$ and $-250<z<250 \mathrm{~cm}$ in the radial $r$ and longitudinal $z$ directions, respectively. These detectors, covering the pseudo-rapidity interval $-0.9 \leq \eta \leq 0.9$ for tracks reaching the outer layer of the TPC, also provide PID information via the specific energy loss $(\mathrm{d} E / \mathrm{d} x)$ measurements.

The measurement of the time of flight of the tracks is based on the TOF detector. On the other hand, the event collision time $t_{\mathrm{ev}}$ is determined with the information coming from both the TOF and the T0 detectors.

The TOF system [4] covers the pseudo-rapidity interval $-0.9 \leq \eta \leq 0.9$ and full azimuthal acceptance. The system is located, according to a cylindrical symmetry, at an average distance of $3.8 \mathrm{~m}$ from the beam pipe spanning an active area of $141 \mathrm{~m}^{2}$. The detector is made of 1593 Multi-gap Resistive Plate Chambers (MRPC), with a sensitive area of $7.4 \times 120 \mathrm{~cm}^{2}$ each. Each MRPC is segmented into 96 readout pads of area $2.5 \times 3.5 \mathrm{~cm}^{2}$. The MRPCs are packed then in five modules for each of the 18 azimuthal sectors of the ALICE spaceframe in a "TOF supermodule", as shown in fig. 1. This detector has a time resolution of $\sim 80$ ps during the data taking [5].

The T0 detector [6] consists of two arrays of Cherenkov counters T0A and T0C, positioned on both sides of the interaction point (IP) at a distance of $374 \mathrm{~cm}$ and $-70 \mathrm{~cm}$ (as shown in fig. 2), covering the pseudorapidity region $4.61 \leq \eta \leq 4.92$ and $-3.28 \leq \eta \leq-2.97$, respectively. The small distance from the IP for T0C had to be chosen because of the space constraints imposed by the front cone of the muon absorber and other forward detectors. On the opposite side the distance of the array T0-A is comfortably far from the congested central region.

\footnotetext{
a e-mail: alice-publications@cern.ch

b See appendix A for the list of collaboration members.
} 


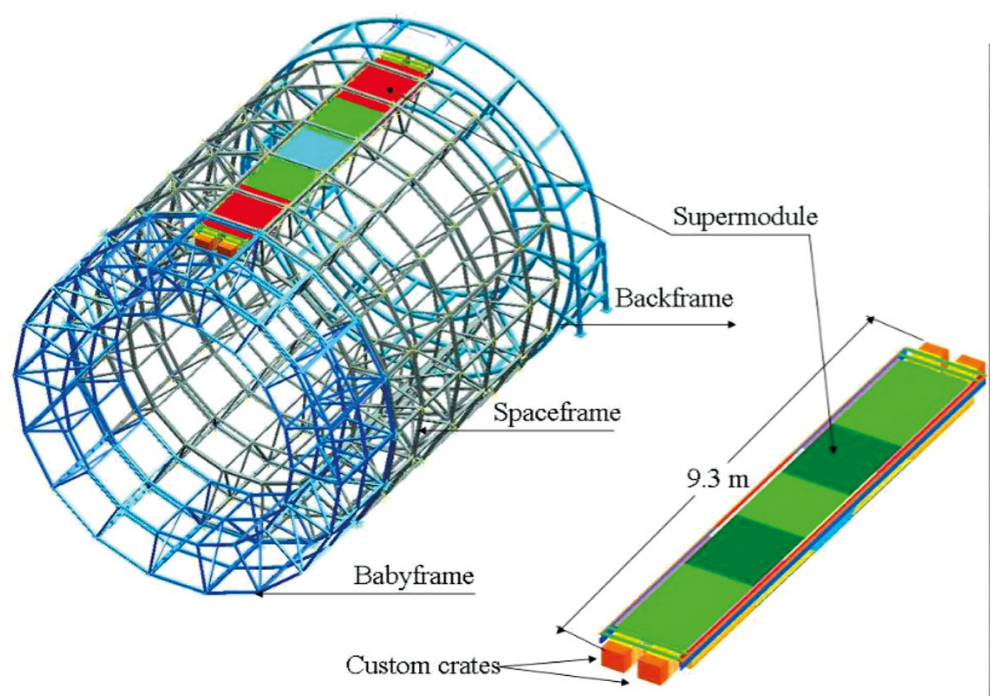

Fig. 1. A schematic layout of one of the 18 TOF supermodules inside the ALICE spaceframe.

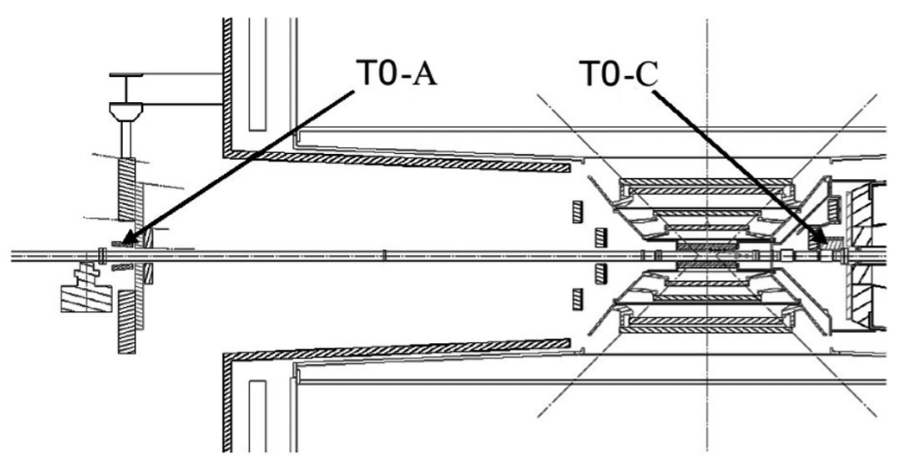

Fig. 2. The layout of the T0 detector arrays inside ALICE.

Each array has 12 cylindrical counters equipped with a quartz radiator $20 \mathrm{~mm}$ in diameter and $20 \mathrm{~mm}$ thick and a photomultiplier tube. The T0 detector provides a measurement of the $t_{\mathrm{ev}}$. It also provides the collision trigger and monitors the luminosity providing fast feedback to the LHC accelerator team. The measured time resolution of the T0 detector is $\sim 50$ ps for single MIP events and reaches $\sim 25 \mathrm{ps}$ at higher multiplicities.

The TOF and the T0 detectors use different front-end electronics but the same digital electronics. The latter is based on the HPTDC (High Performance Time Digital Converter) [7] developed by the CERN Microelectronic Group for LHC experiments. The time measurement is performed with $25 \mathrm{ps}$ bin width resolution with respect to the trigger time, latched with the $40 \mathrm{MHz}$ LHC clock phase. The measurement corresponds for this application to an ionizing particle hit in the TOF MRPC or a photon hit in the T0 photomultipliers. The HPTDC is free running and hit time measurements are stored in internal buffers within a given latency window, waiting for the trigger arrival.

Relevant for the following discussion is also the V0 detector. It consists of two scintillator arrays built around the beam pipe covering the pseudorapidity ranges $2.8 \leq \eta \leq 5.1$ (V0A) and $-3.7 \leq \eta \leq 1.7$ (V0C) and is used for triggering and event selection. In $\mathrm{p}-\mathrm{Pb}$ collisions it is also used to define the multiplicity of the collision exploiting the information from the amplitude of the signal measured by the V0A scintillators [8] while in $\mathrm{Pb}-\mathrm{Pb}$ it is used to define the centrality through the summed amplitudes in the V0 scintillators as described in [9].

The particle identification with the TOF detector is based on the comparison between the time of flight of the track from the primary vertex to the TOF detector and the expected time under a given mass hypothesis $t_{\text {exp }, \mathrm{i}}(\mathrm{i}=\mathrm{e}$, $\left.\mu, \pi, \mathrm{K}, \mathrm{p}, \mathrm{d}, \mathrm{t},{ }^{3} \mathrm{He},{ }^{4} \mathrm{He}\right)$. The former is defined as the difference between the arrival time $t_{\mathrm{TOF}}$ measured by the TOF detector itself and the event collision time $t_{\mathrm{ev}}$. The expected time is the time it would take for a particle of mass $m_{i}$ to go from the interaction point to the TOF. To take into account the energy loss and the consequent variation in the track momentum, $t_{\text {exp }, \mathrm{i}}$ is calculated as the sum of the small time increments $\Delta t_{i, k}$, each of which is the time a particle of mass $m_{i}$ and momentum $p_{k}$ spends to travel along each propagation step $k$ of lenght $\Delta l_{k}$ during the track 
reconstruction procedure:

$$
t_{\mathrm{exp}, \mathrm{i}}=\sum_{k} \Delta t_{i, k}=\sum_{k} \frac{\sqrt{p_{k}^{2}+m_{i}^{2}}}{p_{k}} \Delta l_{k} .
$$

Therefore, the fundamental variable for the TOF PID is $t_{\mathrm{TOF}}-t_{\mathrm{ev}}-t_{\mathrm{exp}, \mathrm{i}}$. Its resolution is

$$
\sigma_{\mathrm{PID}, \mathrm{i}}^{2}=\sigma_{t_{\mathrm{TOF}}}^{2}+\sigma_{t_{\mathrm{ev}}}^{2}+\sigma_{t_{\mathrm{exp}, \mathrm{i}}}^{2}
$$

As mentioned earlier, the TOF detector resolution $\left(\sigma_{t_{\mathrm{TOF}}}\right)$ is $\sim 80$ ps while the uncertainty $\left(\sigma_{t_{\mathrm{exp}, \mathrm{i}}}\right)$ due to the tracking and reconstruction, that includes estimates of the energy losses through the material, depends on the momentum and on the particle species [5]. The uncertainty on the event collision time $\left(\sigma_{t_{\mathrm{ev}}}\right)$ depends on the method used to determine it in the given event.

The simplest PID estimator for a given mass hypothesis $m_{i}$ is then constructed as an $n \sigma$ quantity in the following way:

$$
n \sigma_{\mathrm{TOF}, \mathrm{i}}=\frac{t_{\mathrm{TOF}}-t_{\mathrm{ev}}-t_{\mathrm{exp}, \mathrm{i}}}{\sigma_{\mathrm{PID}, \mathrm{i}}}
$$

This paper focuses on a fundamental term for the TOF PID determination: the event collision time $t_{\mathrm{ev}}$. The methods used for its determination are described in detail in the following sections. Their resolutions, efficiencies and impacts on the PID performance are reported for data samples collected in the different collision systems during RUN 1: pp data at a center-of-mass energy of $\sqrt{s}=7 \mathrm{TeV}, \mathrm{p}-\mathrm{Pb}$ data at a center-of-mass energy per nucleon pair of $\sqrt{s_{\mathrm{NN}}}=5.02 \mathrm{TeV}$ and $\mathrm{Pb}-\mathrm{Pb}$ at $\sqrt{s_{\mathrm{NN}}}=2.76 \mathrm{TeV}$. In sect. 2 the event and track selection are described, in sect. 3 the calibration and timing alignment procedure of the TOF with respect to the LHC clock and in sect. 4 the methods for the determination of the event collision time $t_{\mathrm{ev}}$. Finally, in sect. 5 results for efficiencies, resolutions and impact on PID separation power are presented and discussed. More informations on the general performances of the ALICE detectors in the first period of data taking at LHC are available in [3].

\section{Event and track selection}

For the study reported in this paper the data were selected using a minimum bias trigger based on the V0 detector. Events are further required to have a primary vertex reconstructed either from the tracks reconstructed both in the ITS and in the TPC or from the tracklets, which are track segments built from pairs of hits in the two innermost layers of the ITS. Only events with a reconstructed primary vertex within $10 \mathrm{~cm}$ from the nominal interaction point along the beam directions were used in the analysis. Furthermore, events with multiple reconstructed vertices were rejected, leading to a negligible amount of pile-up events for all the colliding systems [3]. Finally, since the event collision time is a measurement that is needed to identify particles by means of the time-of-flight technique performed by the TOF detector, only events with at least one track associated with a hit in the TOF detector are selected. The number of analyzed events after these cuts is 12 millions for pp at $\sqrt{s}=7 \mathrm{TeV}, 10$ millions for $\mathrm{p}-\mathrm{Pb}$ and 1 million for $\mathrm{Pb}-\mathrm{Pb}$ that are only a subsample of the available statistics collected by ALICE.

The performance of the event collision time will be reported in terms of the TOF track multiplicity of the event, that is the number of tracks associated with a hit on the TOF detector. This choice is driven by the fact that a hit on the TOF is the minimal request that a track has to satisfy to be identified via the time-of-flight procedure. For $\mathrm{Pb}-\mathrm{Pb}$ events, the $t_{\mathrm{ev}}$ measurement performance is also reported in terms of centrality, determined by the sum of the V0 amplitudes and defined in terms of percentiles of the total hadronic $\mathrm{Pb}-\mathrm{Pb}$ cross section [9], while for $\mathrm{p}-\mathrm{Pb}$ in terms of the V0A multiplicity [8].

\section{TOF time alignment and calibration}

As described in [5], the TOF signals are first calibrated for the channel-by-channel offsets (which take into account the differences due to the cable length) and the time-slewing effects. Then, to align the time of flight with respect to the LHC clock, a global shift with respect to the clock phase, $\left\langle t_{\mathrm{ev}}\right\rangle$, is calculated by the TOF itself, for each LHC fill, during the calibration procedure as described below and applied as a global offset to all the measured times.

Due to the fact that the phase of the LHC clock during a fill, as distributed to the experiments, is subject to shifts correlated with the environment temperature (the refractive index of the fibers used for the clock distribution has a dependency on the temperature), $\left\langle t_{\mathrm{ev}}\right\rangle$ is calculated with a five minutes granularity in time. This interval is increased in steps of five minutes if the number of events in the interval is smaller than 1000 or the number of tracks selected for the procedure is smaller than 20000. The time of flight measured for the selected tracks is then compared to the $t_{\text {exp,i }}$ obtained assuming the pion mass hypothesis. The choice of using the pion mass as reference is justified by the 


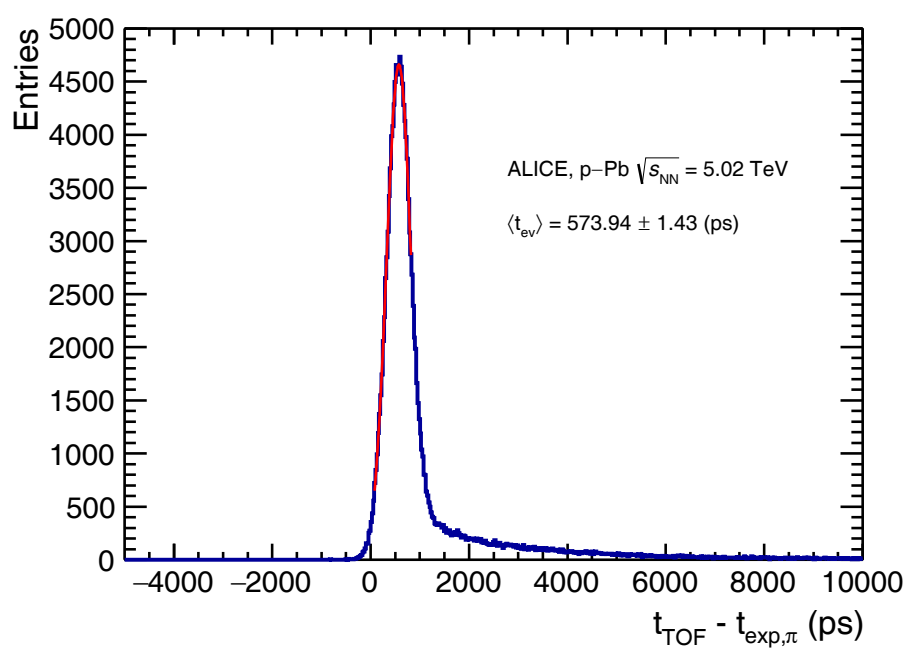

Fig. 3. Average collision time $\left\langle t_{\mathrm{ev}}\right\rangle$ calculated for five minutes of $\mathrm{p}-\mathrm{Pb}$ data taken at $\sqrt{s_{\mathrm{NN}}}=5.02 \mathrm{TeV}$.

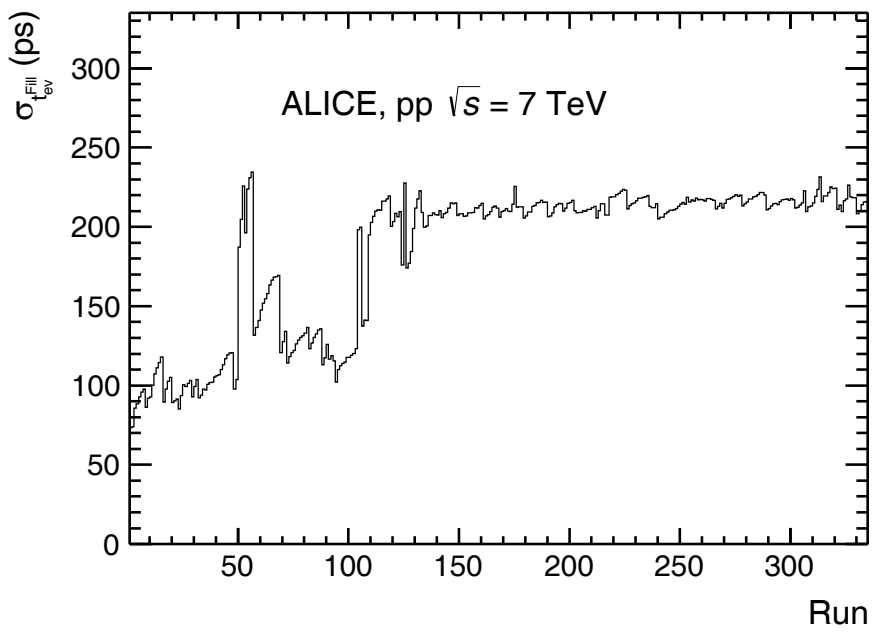

Fig. 4. Resolution of the $t_{\mathrm{ev}}^{\text {Fill }}\left(\sigma_{t_{\mathrm{ev}}^{\mathrm{Fill}}}\right)$ for all $335 \mathrm{pp}$ runs recorded at $\sqrt{s}=7 \mathrm{TeV}$ in 2010 .

fact that pions are the most abundant species produced in the collisions, and they largely dominate the time spectrum distribution. The difference between the measured time of flight and the expected times is fitted with a Gaussian function. Its mean corresponds to the global offset to be applied to all the time-of-flight signals measured in the time interval under study, in order to align the $t_{\mathrm{TOF}}$ with respect to the LHC clock. Figure 3 shows an example of such a fit for $\mathrm{p}-\mathrm{Pb}$ data at $\sqrt{s_{\mathrm{NN}}}=5.02 \mathrm{TeV}$ collected in 2013 .

\section{Methods for the event-by-event collision time determination}

Since the bunches have a small but finite size and it is not known which of the particles in the bunches have collided, the event collision time has a natural spread with respect to the nominal beam crossing. Therefore, an event time $t_{\text {ev }}$ has to be measured on an event-by-event basis. If the event-by-event procedures described below cannot be used, $t_{\text {ev }}$ is set to zero. Conventionally, this null value is named $t_{\mathrm{ev}}^{\text {Fill }}$. It is assumed null because $\left\langle t_{\mathrm{ev}}\right\rangle$ has been already subtracted as part of the calibration procedure described in sect. 3. Its resolution is directly connected to the vertex spread along the beam direction estimated by the ITS per run and derived via $\sigma_{t_{\mathrm{ev}}^{\mathrm{Fill}}}=\sigma_{\mathrm{vertex}} / c$. In fig. 4 the $\sigma_{t_{\mathrm{ev}}^{\mathrm{Fill}}}$ is reported for all the runs of pp collisions at $\sqrt{s}=7 \mathrm{TeV}$ collected during the 2010 data taking.

The variation of $\sigma_{t_{\mathrm{ev}}^{\mathrm{Fill}}}$ shown in fig. 4 depends on the beam optic configurations. After the initial LHC operations $\sigma_{t_{\mathrm{ev}}^{\mathrm{Fill}}}$ became more or less constant at $\sim 200 \mathrm{ps}$. Therefore, if $t_{\mathrm{ev}}$ cannot be computed on an event-by-event basis, $t_{\mathrm{ev}}$ 
is set to $t_{\mathrm{ev}}^{\mathrm{Fill}}$ which has a resolution of $\sim 200 \mathrm{ps}$. This becomes then the dominant term in the TOF PID resolution (see eq. (2)).

To improve the TOF PID performance on an event-by-event basis reducing the $\sigma_{t_{\mathrm{ev}}}$ in eq. (2) with respect to the value of $\sigma_{t_{\mathrm{ev}}^{\mathrm{Fill}}}$, the $t_{\mathrm{ev}}$ can be computed by the TOF itself $\left(t_{\mathrm{ev}}^{\mathrm{TOF}}\right)$, by the T0 detector $\left(t_{\mathrm{ev}}^{\mathrm{T} 0}\right)$ or by a combination of the two $\left(t_{\mathrm{ev}}^{\text {Best }}\right)$ as shown in the following sections.

\subsection{Event collision time measurement performed by the TOF detector}

The event collision time is estimated by the TOF detector $\left(t_{\mathrm{ev}}^{\mathrm{TOF}}\right)$ on an event-by-event basis by means of a $\chi^{2}$ minimization procedure. Having in the event $n_{\text {tracks }}$ matched to a corresponding hit on the TOF detector and satisfying basic quality cuts, it is possible to define certain combinations of masses $\vec{m}_{i}$ assigning independently for each track the $\pi, \mathrm{K}$ or $\mathrm{p}$ mass. The index $i$ indicates one of the possible combination $\left(m_{1}, m_{2}, \ldots, m_{n_{\text {tracks }}}\right)$ among the $3^{n_{\text {tracks }}}$ ones.

For each track the following weight is evaluated

$$
W_{i}=\frac{1}{\sigma_{\mathrm{TOF}}^{2}+\sigma_{t_{\mathrm{exp}, \mathrm{i}}}^{2}} .
$$

The event time is then deduced as in eq. (5) where the track index is omitted for simplicity,

$$
t_{\mathrm{ev}}^{\mathrm{TOF}}\left(\vec{m}_{i}\right)=\frac{\sum_{n_{\text {tracks }}} W_{i}\left(t_{\mathrm{TOF}}-t_{\mathrm{exp}, \mathrm{i}}\right)}{\sum_{n_{\text {tracks }}} W_{i}},
$$

and the resolution is given by

$$
\sigma_{t_{\mathrm{ev}}^{\mathrm{TOF}}}\left(\vec{m}_{i}\right)=\sqrt{\frac{1}{\sum_{n_{\text {tracks }}} W_{i}}}
$$

The following $\chi^{2}$ is then calculated

$$
\chi^{2}\left(\vec{m}_{i}\right)=\sum_{n_{\text {tracks }}} \frac{\left(\left(t_{\mathrm{TOF}}-t_{\mathrm{ev}}^{\mathrm{TOF}}\left(\vec{m}_{i}\right)\right)-t_{\mathrm{exp}, \mathrm{i}}\right)^{2}}{\sigma_{\mathrm{TOF}}^{2}+\sigma_{t_{\mathrm{exp}, \mathrm{i}}}^{2}} .
$$

The combination $\vec{m}_{i}$ that minimizes this $\chi^{2}$ is used to derive $t_{\text {ev }}^{\text {TOF }}$ via eq. $(5)$.

This general procedure is refined in two ways. To avoid possible PID biases which are important especially in low multiplicity events, a track cannot be used to compute the $t_{\mathrm{ev}}^{\mathrm{TOF}}$ to perform PID on the track itself. This means that, in principle, each track has to be removed by the sample before calculating the $t_{\mathrm{ev}}^{\mathrm{TOF}}$, repeating this procedure for each track. This approach would result in an excessive request of computing resources when the number of tracks is large. Therefore, in order to optimize the procedure, the tracks are divided into ten momentum intervals. The $t_{\mathrm{ev}}^{\mathrm{TOF}}$ is calculated for each momentum interval using only the tracks belonging to the other nine momentum bins. With this procedure the $t_{\mathrm{ev}}^{\mathrm{TOF}}$ to be used in eq. (3) to perform PID on a track is not biased by the implicit identification of the track performed by the $t_{\mathrm{ev}}$ algorithm with the TOF and is evaluated using only the tracks in the momentum bins other than the one the track belongs to. Finally, to avoid an excessive computational load due to the combinatorics, this evaluation is done dividing the sample of tracks in the event in several subsamples and the weighted average of the results is then taken.

It should be noted that $\sigma_{t_{\mathrm{ev}}^{\mathrm{TOF}}}$ is dependent on the event track multiplicity because, according to eq. (6) it scales as $\sim 1 / \sqrt{n_{\text {tracks }}}$.

\subsection{Event collision time measurement performed by the T0 detector}

The T0 detector can provide two time measurements, $t_{\mathrm{T} 0 \mathrm{~A}}$ and $t_{\mathrm{T} 0 \mathrm{C}}$, one for each of its two sub-detectors T0A and T0C, corresponding to the fastest signals among its photomultipliers. When both values are available, the event collision time is defined as $t_{\mathrm{ev}}^{\mathrm{TOAC}}=\left(t_{\mathrm{T} 0 \mathrm{~A}}+t_{\mathrm{T} 0 \mathrm{C}}\right) / 2$, which is independent of the event vertex position. In low multiplicity events, when only one of the two arrays of Cherenkov counters produces a signal, $t_{\mathrm{T} 0 \mathrm{~A}}$ or $t_{\mathrm{T} 0 \mathrm{C}}$ can be used as a measurement of the event collision time once a correction for the z-position of the primary vertex (as measured by the ITS with an accuracy of $50 \mu \mathrm{m})$ is taken into account.

The time resolution of the T0 detector [3] is related to the number of photoelectrons emitted from the photocathode of each PMT. This, in turn, is directly proportional to the number of MIPs traversing the quartz radiator. In principle it would be possible to estimate the resolution for each event based on the registered amplitude in each T0 module 


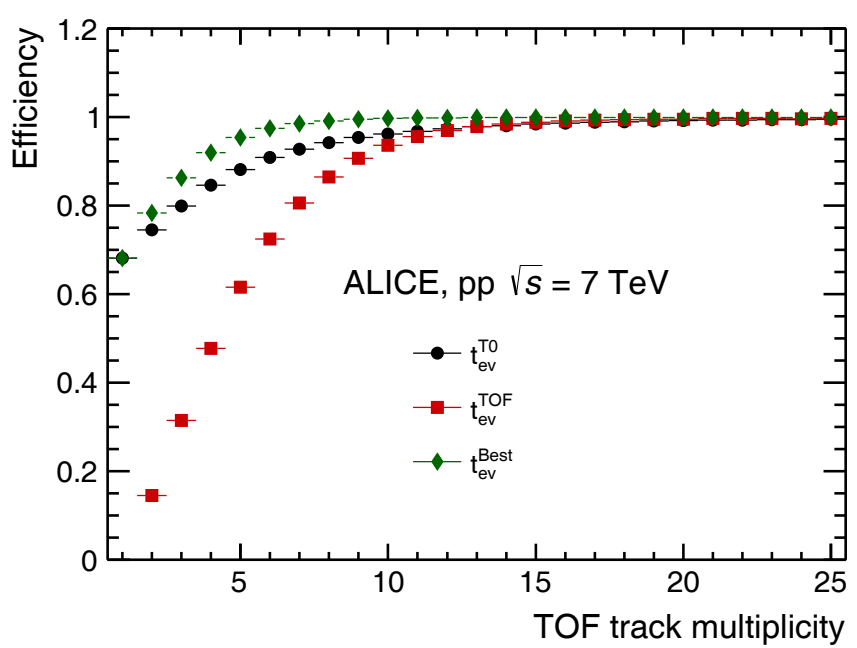

Fig. 5. Efficiency of the $t_{\mathrm{ev}}^{\mathrm{TO}}$ (circles), $t_{\mathrm{ev}}^{\mathrm{TOF}}$ (squares) and $t_{\mathrm{ev}}^{\mathrm{Best}}$ (diamond) as a function of the TOF track multiplicity for pp collisions at $\sqrt{s}=7 \mathrm{TeV}$.

but the analysis procedures implemented during RUN 1 yielded only the average value per run. As a consequence the time resolution depends on the average multiplicity of the events in the run and hence on the colliding system. At the moment, the small dependence of $\sigma_{t_{\mathrm{ev}}^{\mathrm{To}}}$ on the track multiplicity is not taken into account since it is only of the order of a maximum of $20 \%$, negligible when compared to the dependence of $\sigma_{t_{\mathrm{ev}}^{\mathrm{TO}}}$ on the TOF track multiplicity as will be shown later, and smaller than the run by run fluctuation. When both $t_{\mathrm{T} 0 \mathrm{~A}}$ and $t_{\mathrm{T} 0 \mathrm{C}}$ measurements are available the resolution can be estimated by the width of the $\left(t_{\mathrm{T} 0 \mathrm{~A}}-t_{\mathrm{T} 0 \mathrm{C}}\right) / 2$ distribution after both $t_{\mathrm{T} 0 \mathrm{~A}}$ and $t_{\mathrm{T} 0 \mathrm{C}}$ are corrected for the vertex position. In $\mathrm{Pb}-\mathrm{Pb}$ and $\mathrm{pp}$ collisions the resolutions are $\sigma_{t_{\mathrm{ev}}^{\mathrm{TOAC}}} \sim 25$ and $\sigma_{t_{\mathrm{ev}}^{\mathrm{TOAC}}} \sim 50 \mathrm{ps}$, respectively. The difference is due to the different average multiplicity of the events in the two colliding systems and the resulting different signal amplitudes. When only $t_{\mathrm{T} 0 \mathrm{~A}}$ or $t_{\mathrm{T} 0 \mathrm{C}}$ are available, the resolutions are $\sigma_{t_{\mathrm{ev}}^{\mathrm{TOA}}} \sim 50 \mathrm{ps}$ and $\sigma_{t_{\mathrm{ev}}^{\mathrm{TOC}}} \sim 30 \mathrm{ps}$ in $\mathrm{Pb}-\mathrm{Pb}$ collisions and $\sigma_{t_{\mathrm{ev}}^{\mathrm{TOA}}} \sim 100 \mathrm{ps}$ and $\sigma_{t_{\mathrm{ev}}^{\mathrm{ToC}}} \sim 60 \mathrm{ps}$ in pp collisions. The difference is due to the different distance of T0A and T0C from the interaction point.

To reach this time resolution, an accurate calibration procedure for T0 is needed. Before every data taking period, gain and slewing corrections are determined using a set of laser runs, where the laser intensity is varied. The mean time value for each photomultiplier, after slewing correction, is optimized for the minimum bias trigger for each run.

\subsection{Combination of the TOF and T0 measurements}

For each event, $t_{\mathrm{ev}}$ is obtained combining in a single estimation $\left(t_{\mathrm{ev}}^{\text {Best }}\right)$ the results from the different methods available.

If the $t_{\mathrm{ev}}$ measurement can be provided by only TOF or T0 detector, $t_{\mathrm{ev}}^{\mathrm{Best}}$ will correspond, respectively, to $t_{\mathrm{ev}}^{\mathrm{TOF}}$ or $t_{\mathrm{ev}}^{\mathrm{T} 0}$. If both of them are available than $t_{\mathrm{ev}}^{\mathrm{Best}}$ is estimated by their weighted mean where the weights are the inverse of the square of the resolutions. If both methods are not available, $t_{\mathrm{ev}}^{\mathrm{Best}}$ fails and $t_{\mathrm{ev}}$ is defined by the $t_{\mathrm{ev}}^{\text {Fill }}$. In the last case, the resolution is $\sim 200$ ps.

The relative occurrence and resolutions of these three cases depend on the multiplicity of the event and therefore, indirectly, on the collision type, as will be shown in sect. 5 .

\section{Results}

Results related to the efficiency of the methods used to define the event collision time as a function of the TOF track multiplicity, their resolution and their impact on the PID performance are reported in this section. For p-Pb and $\mathrm{Pb}-\mathrm{Pb}$ collision systems the analysis is provided also as a function of the multiplicity class or centrality of the collision.

\subsection{Efficiency of the determination of $t_{e v}^{T O F}, t_{e v}^{T 0}$ and $t_{e v}^{B e s t}$}

In fig. 5 the efficiency of the determination of $t_{\mathrm{ev}}^{\mathrm{TOF}}, t_{\mathrm{ev}}^{\mathrm{T} 0}$ and $t_{\mathrm{ev}}^{\mathrm{Best}}$ is reported as a function of the TOF track multiplicity in pp collisions at $\sqrt{s}=7 \mathrm{TeV}$. 

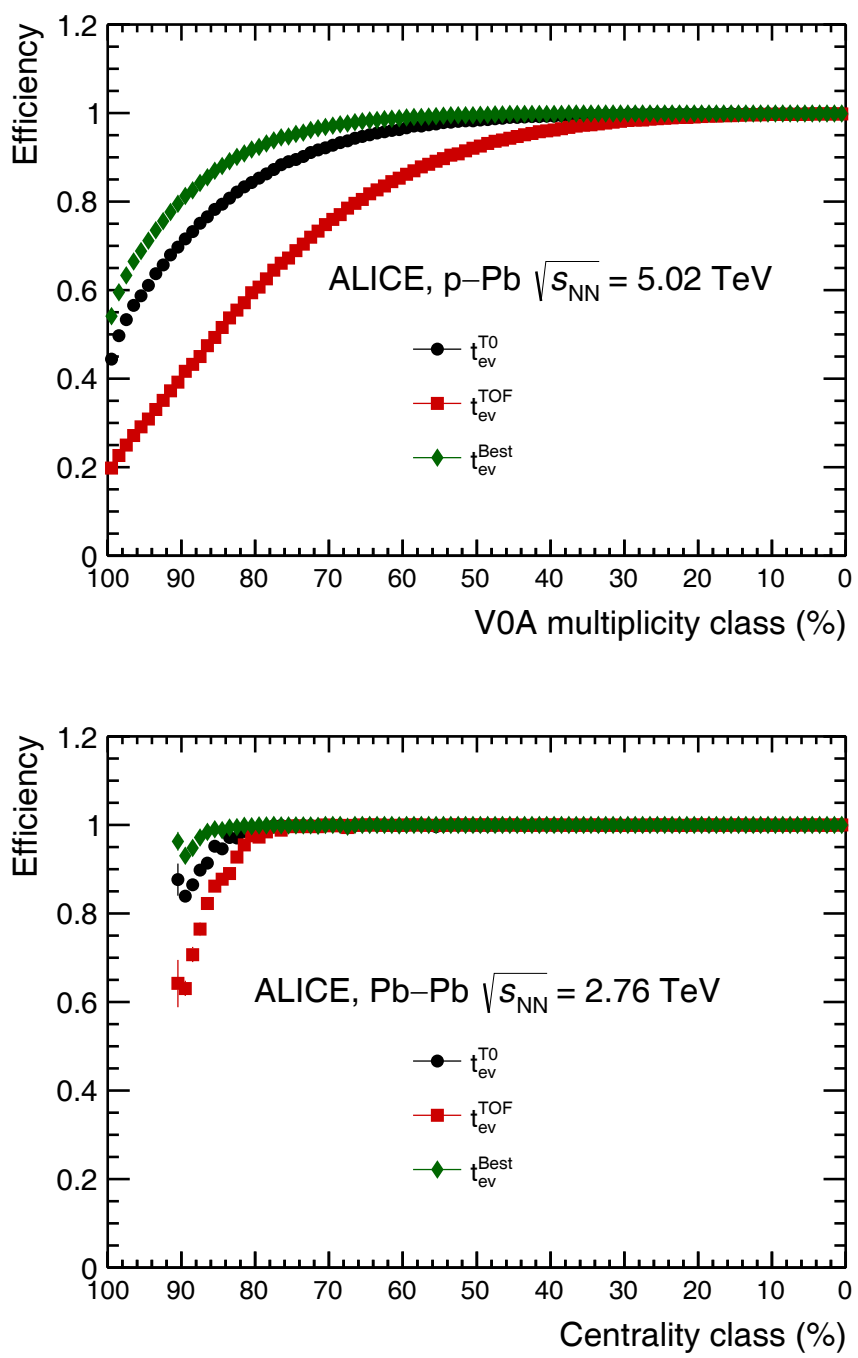

Fig. 6. Efficiencies of the methods $t_{\mathrm{ev}}^{\mathrm{T} 0}$ (circles), $t_{\mathrm{ev}}^{\mathrm{TOF}}$ (squares) and $t_{\mathrm{ev}}^{\text {Best }}$ (diamond) as a function of the V0A multiplicity class for $\mathrm{p}-\mathrm{Pb}$ collisions at $\sqrt{s}=2.76 \mathrm{TeV}$ (top) and of the V0M Centrality class for Pb-Pb collisions $\sqrt{s_{\mathrm{NN}}}=2.76 \mathrm{TeV}$ (bottom).

The efficiency is defined as the fraction of events for which the $t_{\mathrm{ev}}^{\mathrm{TOF}}, t_{\mathrm{ev}}^{\mathrm{T} 0}$ or $t_{\mathrm{ev}}^{\mathrm{Best}}$ has been measured compared to the ones selected as explained in sect. 1. Since $t_{\mathrm{ev}}^{\mathrm{TOF}}$ and thus $t_{\mathrm{ev}}^{\text {Best }}$ are defined in ten momentum bins (see sect. 4.1) they are considered efficient if the measurement is available in at least one momentum bin.

The TOF track multiplicity of the event is the number of tracks matched with a hit on the TOF detector that is the number of tracks with an associated time-of-flight measurement. This is the minimal request for a track to be identified by the time-of-flight method. It is important to notice that the TOF track multiplicity does not represent the number of tracks that are used by the TOF algorithm to compute the $t_{\mathrm{ev}}^{\mathrm{TOF}}$, that is actually slightly lower since in the algorithm a further basic selection on the quality of the track is applied to guarantee a good quality of the $t_{\mathrm{ev}}^{\mathrm{TOF}}$. What is reported in fig. 5 is, therefore, not the algorithmic efficiency.

From sect. 4.1 it is evident that the minimum number of tracks to compute $t_{\mathrm{ev}}^{\mathrm{TOF}}$ is two. Therefore the $t_{\mathrm{ev}}^{\mathrm{TOF}}$ efficiency in the first bin is not shown in fig. 5 .

In pp collisions, for very low multiplicity events, the T0 detector can provide a $t_{\mathrm{ev}}$ measurement with an efficiency of the order of $\sim 70 \%$ that increases with the track multiplicity. At the same time, for all events having high multiplicity, the $t_{\mathrm{ev}}^{\mathrm{TOF}}$ method is able to provide a $t_{\mathrm{ev}}$ measurement.

The curve corresponding to $t_{\mathrm{ev}}^{\text {Best }}$ shows how the two techniques can be combined to minimize the number of events, in particular at low multiplicity, where an event-by-event $t_{\mathrm{ev}}$ measurement cannot be provided and only $t_{\mathrm{ev}}^{\text {Fill }}$ is available. In pp collisions at $\sqrt{s}=7 \mathrm{TeV}$, when more than three tracks reach the TOF the event time efficiency is greater than $80 \%$.

In fig. 6 the efficiency of the $t_{\mathrm{ev}}^{\mathrm{T} 0}, t_{\mathrm{ev}}^{\mathrm{TOF}}$ and $t_{\mathrm{ev}}^{\mathrm{Best}}$ is reported as a function of the $\mathrm{V} 0 \mathrm{~A}$ multiplicity class in $\mathrm{p}-\mathrm{Pb}$ and centrality in $\mathrm{Pb}-\mathrm{Pb}$ collisions, respectively. 
Table 1. Fraction of events (percentage) for which the $t_{\mathrm{ev}}^{\mathrm{TOF}}$ and $t_{\mathrm{ev}}^{\mathrm{TO}}$ can be provided when explicitly requested. The results are shown for pp collisions at $\sqrt{s}=7 \mathrm{TeV}, \mathrm{p}-\mathrm{Pb}$ collisions at $\sqrt{s_{\mathrm{NN}}}=5.02 \mathrm{TeV}$ and $\mathrm{Pb}-\mathrm{Pb}$ collisions at $\sqrt{s_{\mathrm{NN}}}=2.76 \mathrm{TeV}$.

\begin{tabular}{|c|c|c|c|c|}
\hline & $t_{\mathrm{ev}}^{\mathrm{TOF}}(\%)$ & \multicolumn{3}{|c|}{$t_{\mathrm{ev}}^{\mathrm{T} 0}(\%)$} \\
\hline \hline & & $t_{\mathrm{ev}}^{\mathrm{T} 0 \mathrm{~A}}$ & $t_{\mathrm{ev}}^{\mathrm{TOC}}$ & $t_{\mathrm{ev}}^{\mathrm{TOAC}}$ \\
\hline \hline $\mathrm{pp} \sqrt{s}=7 \mathrm{TeV}$ & 52.5 & 18.0 & 21.8 & 45.2 \\
\hline $\mathrm{p}-\mathrm{Pb} \sqrt{s_{\mathrm{NN}}}=5.02 \mathrm{TeV}$ & 81.8 & 13.0 & 11.0 & 68.4 \\
\hline & 81.8 & \multicolumn{3}{|c|}{$\sum=92.4$} \\
\hline $\mathrm{Pb}-\mathrm{Pb} \sqrt{s_{\mathrm{NN}}}=2.76 \mathrm{TeV}$ & 99.6 & 0.3 & 0.5 & 98.9 \\
\hline & 99.6 & \multicolumn{3}{|c|}{$\sum=99.7$} \\
\hline
\end{tabular}

Table 2. Fraction of events (percentage) for which the $t_{\mathrm{ev}}^{\text {Best }}$ can be provided when explicitly requested (total and for each subcase). The results are shown for pp at $\sqrt{s}=7 \mathrm{TeV}, \mathrm{p}-\mathrm{Pb}$ collisions at $\sqrt{s_{\mathrm{NN}}}=5.02 \mathrm{TeV}$ and $\mathrm{Pb}-\mathrm{Pb}$ collisions at $\sqrt{s_{\mathrm{NN}}}=$ $2.76 \mathrm{TeV}$

\begin{tabular}{|c|c|c|c|c|c|c|c|c|}
\hline & \multicolumn{7}{|c|}{$t_{\mathrm{ev}}^{\text {Best }}(\%)$} & \multirow[b]{2}{*}{$t_{\mathrm{ev}}^{\mathrm{Fill}}$} \\
\hline & $t_{\mathrm{ev}}^{\mathrm{TOF}}$ & $t_{\mathrm{ev}}^{\mathrm{T} 0 \mathrm{~A}}$ & $t_{\mathrm{ev}}^{\mathrm{TOF}+\mathrm{T} 0 \mathrm{~A}}$ & $t_{\mathrm{ev}}^{\mathrm{T} 0 \mathrm{C}}$ & $t_{\mathrm{ev}}^{\mathrm{TOF}+\mathrm{T} 0 \mathrm{C}}$ & $t_{\mathrm{ev}}^{\mathrm{T} 0 \mathrm{AC}}$ & $t_{\mathrm{ev}}^{\mathrm{TOF}+\mathrm{T} 0 \mathrm{AC}}$ & \\
\hline \multirow[t]{2}{*}{$\mathrm{pp} \sqrt{s}=7 \mathrm{TeV}$} & 4.0 & 10.8 & 7.2 & 11.5 & 10.3 & 14.2 & 31.0 & 11.0 \\
\hline & \multicolumn{7}{|c|}{$\sum=89.0$} & \\
\hline \multirow[t]{2}{*}{$\mathrm{p}-\mathrm{Pb} \sqrt{s_{\mathrm{NN}}}=5.02 \mathrm{TeV}$} & 2.9 & 4.2 & 8.8 & 4.0 & 7.0 & 5.4 & 63.0 & 4.7 \\
\hline & \multicolumn{7}{|c|}{$\sum=95.3$} & \\
\hline \multirow[t]{2}{*}{$\mathrm{Pb}-\mathrm{Pb} \sqrt{s_{\mathrm{NN}}}=2.76 \mathrm{TeV}$} & 0.2 & 0.09 & 0.2 & 0.1 & 0.4 & 0.1 & 98.8 & 0.1 \\
\hline & \multicolumn{7}{|c|}{$\sum=99.9$} & \\
\hline
\end{tabular}

In $\mathrm{p}-\mathrm{Pb}$ collisions, from 0 to $40 \%$ V0A multiplicity class, both the T0 and the TOF are fully efficient in determining the collision time. For more peripheral events the T0 detector has the highest efficiency in providing a $t_{\mathrm{ev}}$ measurement. For $\mathrm{Pb}-\mathrm{Pb}$ collisions only for the most peripheral events (centrality $>80 \%$ ) the T0 has an efficiency higher than the TOF. In $\mathrm{Pb}-\mathrm{Pb}$ collisions the $t_{\mathrm{ev}}^{\text {Best }}$ is $100 \%$ efficient except for the very peripheral events. As a consequence, the $t_{\mathrm{ev}}^{\text {Fill }}$ is basically never used. It is worth to notice that the efficiency curves would have similar trend than the ones in fig. 5 once plotted as a function of the TOF track multiplicity instead of the V0A multiplicity class or centrality since the efficiency mainly depends on the track multiplicity.

The overall efficiency defined as the fraction (in percentage) of events with at least one track associated to a hit in the TOF detector for which the $t_{\mathrm{ev}}^{\mathrm{T} 0}, t_{\mathrm{ev}}^{\mathrm{TOF}}$ and $t_{\mathrm{ev}}^{\mathrm{Best}}$ can be provided, is reported in table 1 and table 2 . The first column of table 1 represents the fraction of events (in \%) for which the $t_{\mathrm{ev}}^{\mathrm{TOF}}$ can be provided in at least one momentum bin. It can be seen that in pp at $\sqrt{s}=7 \mathrm{TeV} t_{\mathrm{ev}}^{\mathrm{TOF}}$ is measured only in less than $53 \%$ of events. This percentage increases reaching $99.6 \%$ in $\mathrm{Pb}-\mathrm{Pb}$ collisions. The second column shows the fraction of events (in \%) for which the $t_{\mathrm{ev}}^{\mathrm{T} 0}$ can be provided. In this case, if both T0A and T0C provide a signal, the $t_{\mathrm{ev}}^{\mathrm{T} 0 \mathrm{AC}}$ is used otherwise the individual $t_{\mathrm{ev}}^{\mathrm{T}} \mathrm{A}$ or $t_{\mathrm{ev}}^{\mathrm{T} 0 \mathrm{C}}$ are used. From pp at $\sqrt{s}=7 \mathrm{TeV}$ to $\mathrm{Pb}-\mathrm{Pb}$ at $\sqrt{s_{\mathrm{NN}}}=2.76 \mathrm{TeV}$ the efficiency of the $t_{\mathrm{ev}}^{\mathrm{TO}}$ increases from $85 \%$ to $99.7 \%$ with an increase of the efficiency of the $t_{\mathrm{ev}}^{\mathrm{TOAC}}$ as expected. The fraction of events for which only the T0A or T0C is used decreases.

In table 2 the efficiency (in \%) of the $t_{\text {ev }}^{\text {Best }}$ also for each exclusive subcases is reported. The outcomes of the possible combinations resulting in a $t_{\mathrm{ev}}^{\text {Best }}$ measurement are detailed in the seven subcolumns. In $\mathrm{Pb}-\mathrm{Pb}$ collisions, for most of the events both $t_{\mathrm{ev}}^{\mathrm{TOF}}$ and $t_{\mathrm{ev}}^{\mathrm{T} 0}$ are available.

\subsection{Resolution of the $t_{\mathrm{ev}}^{\text {TOF }}$ and $\mathrm{t}_{\mathrm{ev}}^{\text {Best }}$ as a function of the TOF track multiplicity}

In fig. 7 (top) the $t_{\mathrm{ev}}^{\mathrm{TOF}}$ resolution $\left(\sigma_{t_{\mathrm{ev}}^{\mathrm{TOF}}}\right)$ is shown as a function of the TOF track multiplicity for pp data at $\sqrt{s}=7 \mathrm{TeV}, \mathrm{p}-\mathrm{Pb}$ collisions at $\sqrt{s_{\mathrm{NN}}}=5.02 \mathrm{TeV}$ and $\mathrm{Pb}-\mathrm{Pb}$ collisions at $\sqrt{s_{\mathrm{NN}}}=2.76 \mathrm{TeV}$.

The trend with the multiplicity is the same for all the data sets since $\sigma_{t_{\mathrm{ev}}^{\mathrm{ToF}}}$ mainly depends on the number of tracks used by the algorithm that is related in turn to the TOF track multiplicity. 

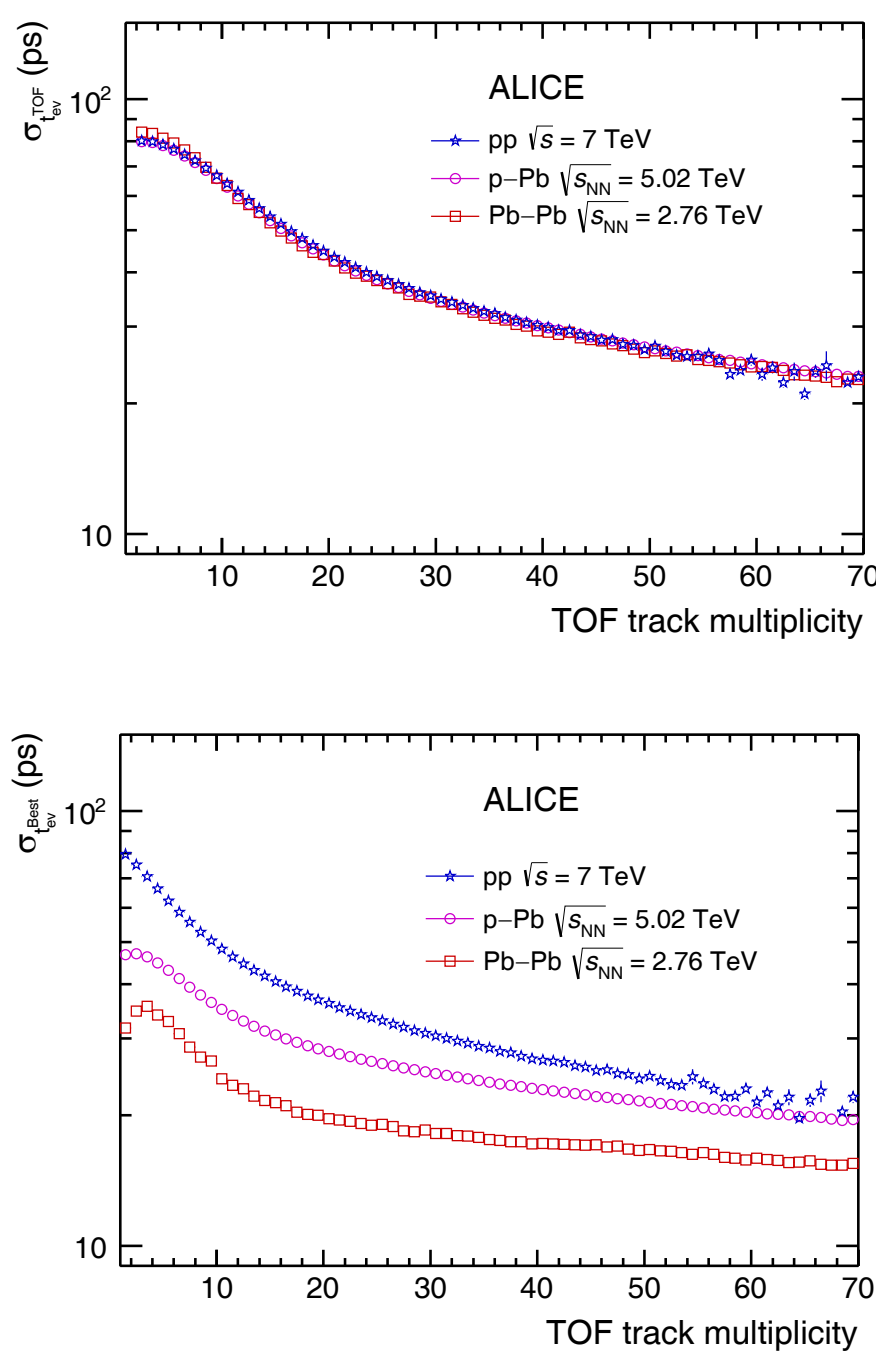

Fig. 7. Resolution of $t_{\mathrm{ev}}^{\mathrm{TOF}}$ (top) and $t_{\mathrm{ev}}^{\text {Best }}$ (bottom) as a function of the TOF track multiplicity for pp collisions at $\sqrt{s}=7 \mathrm{TeV}$ (star), p-Pb collisions at $\sqrt{s_{\mathrm{NN}}}=5.02 \mathrm{TeV}$ (circle) and $\mathrm{Pb}-\mathrm{Pb}$ collisions at $\sqrt{s_{\mathrm{NN}}}=2.76 \mathrm{TeV}$ (square). As a reference, TOF track multiplicity $=15$ corresponds to $50 \%$ V0A multiplicity class in p- $\mathrm{Pb}$ and $80 \%$ centrality class in $\mathrm{Pb}-\mathrm{Pb}$.

The resolution improves from $\sim 80 \mathrm{ps}$ in low multiplicity events, to $20 \mathrm{ps}$ for high multiplicity events. As a consequence, $\sigma_{t_{\mathrm{ev}}^{\mathrm{TOF}}}$ is a significant contribution of the TOF PID resolution $\sigma_{\text {PID }}$ reported in eq. (2) only for low multiplicity events, when it is of the same order of the TOF resolution $\sigma_{t_{\mathrm{TOF}}}$. It becomes negligible at higher track multiplicities. While the resolution as a function of multiplicity is the same for the different colliding systems, it is important to remind here that what is different is the overall fraction of events for which the $t_{\mathrm{ev}}^{\mathrm{TOF}}$ can be provided as can be seen in table 1. It depends on the mean multiplicity of the events that increases from pp to $\mathrm{p}-\mathrm{Pb}$ and to $\mathrm{Pb}-\mathrm{Pb}$ collisions.

In fig. 7 (bottom) the resolution of $t_{\mathrm{ev}}^{\text {Best }}\left(\sigma_{t_{\mathrm{ev}} \text { Best }}\right)$ is reported as a function of the TOF track multiplicity for pp collisions at $\sqrt{s}=7 \mathrm{TeV}$, for p- $\mathrm{Pb}$ and $\mathrm{Pb}-\mathrm{Pb}$ data at $\sqrt{s_{\mathrm{NN}}}=5.02 \mathrm{TeV}$ and $\sqrt{s_{\mathrm{NN}}}=2.76 \mathrm{TeV}$, respectively. It depends on two main factors: the track multiplicity and the colliding system. The first defines the $t_{\mathrm{ev}}^{\mathrm{TOF}}$ resolution while the second the $\sigma_{t_{\mathrm{ev}}^{\mathrm{To}}}$ that decreases moving from pp to $\mathrm{p}-\mathrm{Pb}$ to $\mathrm{Pb}-\mathrm{Pb}$ since, as explained before, $\sigma_{t_{\mathrm{e}}^{\mathrm{To}}}$ depends only on the mean event multiplicity being defined per run and not per event. The exclusive probability of the seven possible subcases of $t_{\mathrm{ev}}^{\text {Best }}$ plays a role here in particular to explain the pattern observed at low multiplicity in fig. 7 for the $\mathrm{Pb}-\mathrm{Pb}$ case.

In fig. 8 the efficiency as a function of the TOF track multiplicity of the possible outcomes of the $t_{\mathrm{ev}}^{\text {Best }}$ are shown for $\mathrm{Pb}-\mathrm{Pb}$ collisions at $\sqrt{s_{\mathrm{NN}}}=2.76 \mathrm{TeV}$. 


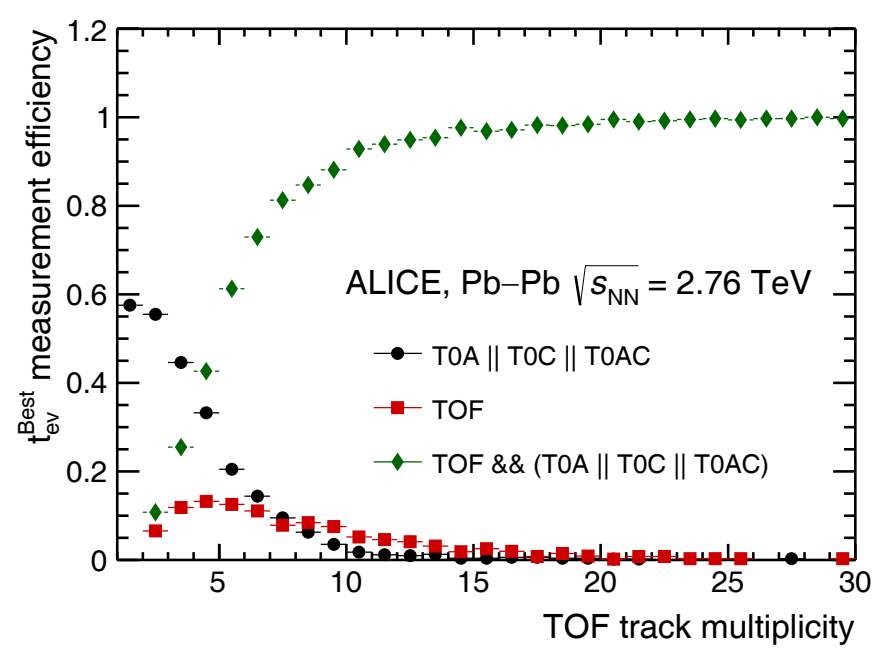

Fig. 8. Fraction of events for which the $t_{\mathrm{ev}}^{\mathrm{Best}}$ is provided exclusively by the T0, no matter if T0A, T0C or T0AC (circle), or exclusively by the TOF (square) or a combination of the two (green) in $\mathrm{Pb}-\mathrm{Pb}$ collisions at $\sqrt{s_{\mathrm{NN}}}=2.76 \mathrm{TeV}$.

It is evident that, for less than 3 tracks matched to the TOF, for most of the events the $t_{\mathrm{ev}}^{\text {Best }}$ is provided by the T0 while, increasing the multiplicity, the combination of the T0 and TOF measurements becomes the dominant term. The interplay of all these factors define the shape of the $\sigma_{t_{\mathrm{ev}}^{\mathrm{Best}}}$ reported in the bottom plot of fig. 7 .

\subsection{Effect of the $t_{e v}$ resolution on the PID performance}

In this section, the impact on the PID performance due to the different methods used for the event collision time determination is assessed. This is studied via the $\mathrm{K}-\pi$ and $\mathrm{p}-\mathrm{K}$ separation power: $n \sigma_{i, j}\left(t_{\mathrm{ev}}^{k}\right)=\left(t_{\exp , \mathrm{i}}-t_{\mathrm{exp}, \mathrm{j}}\right) / \sigma_{\mathrm{PID}, \mathrm{j}}\left(t_{\mathrm{ev}}^{k}\right)$, where $\mathrm{i}, \mathrm{j}=\pi, \mathrm{K}, \mathrm{p}$ and $\sigma_{\mathrm{PID}, \mathrm{j}}^{2}\left(t_{\mathrm{ev}}^{k}\right)=\sigma_{\mathrm{TOF}}^{2}+\sigma_{t_{\mathrm{ev}}}^{2}+\sigma_{t_{\mathrm{exp}, \mathrm{j}}}^{2}$ with $k=\mathrm{TOF}, \mathrm{T} 0$, Best and Fill.

In fig. $9, n \sigma_{\mathrm{K}, \pi}\left(t_{\mathrm{ev}}^{k}\right)$ and $n \sigma_{\mathrm{p}, \mathrm{K}}\left(t_{\mathrm{ev}}^{k}\right)$ are shown as a function of the transverse momentum of the track.

The separation power does not significantly change when changing the $t_{\mathrm{ev}}$ estimator $\left(t_{\mathrm{ev}}^{\mathrm{TOF}}, t_{\mathrm{ev}}^{\mathrm{T} 0}\right.$ or $\left.t_{\mathrm{ev}}^{\mathrm{Best}}\right)$. On the other hand, it gets worse if the $t_{\mathrm{ev}}^{\mathrm{Fill}}$ is used since its resolution is much worse than the one of all the others. If a three sigma separation is requested, the $\pi-\mathrm{K}$ separation is achievable only up to $1.3 \mathrm{GeV} / c$ instead of up to $2 \mathrm{GeV} / c$ if the $t_{\mathrm{ev}}^{\text {Fill }}$ is used and the K-p separation can be defined only up to $2.2 \mathrm{GeV} / c$ instead of up to $3.5 \mathrm{GeV} / c$.

\section{Conclusions}

The determination of the event collision time in ALICE is needed to perform particle identification in the intermediate region of momentum $(0.5-4.0 \mathrm{GeV} / c)$ with the time-of-flight method. It can be provided on an event-by-event basis by the T0 detector $\left(t_{\mathrm{ev}}^{\mathrm{T} 0}\right)$ or the TOF detector itself $\left(t_{\mathrm{ev}}^{\mathrm{TOF}}\right)$. When both the measurements are available a weighted mean can be defined $\left(t_{\mathrm{ev}}^{\mathrm{Best}}\right)$. In case none of the previous methods can be used, mainly for low multiplicity events, only an average collision time $\left(t_{\mathrm{ev}}^{\text {Fill }}\right)$ can be considered, with a resolution of $\sim 200 \mathrm{ps,} \mathrm{which} \mathrm{worsens} \mathrm{the} \mathrm{TOF} \mathrm{PID}$ performance. In this paper the methods for the event collision time determination in ALICE have been reviewed, together with their performance during LHC RUN 1 data in terms of efficiency, resolution and impact on the TOF PID.

It has been shown how, for very low multiplicity events, the T0 detector plays a crucial role since it has a higher efficiency in providing $t_{\mathrm{ev}}$ when compared to the TOF detector. For example, when five tracks reach the TOF, the $t_{\mathrm{ev}}^{\mathrm{T} 0}$ efficiency is $\sim 85 \%$ compared to the $60 \%$ of the TOF detector. The $t_{\mathrm{ev}}^{\mathrm{TOF}}$ efficiency increases with the rise of the track multiplicity reaching $\sim 100 \%$ when 15 tracks reach the TOF.

In the analysed data set and given the current level of calibration of detectors, for high multiplicity events the resolution of the event collision time becomes a negligible term in the time-of-flight resolution. This is achieved combining the $t_{\mathrm{ev}}^{\mathrm{TOF}}$ and $t_{\mathrm{ev}}^{\mathrm{T} 0}$ measurements. In pp collisions at $\sqrt{s}=7 \mathrm{TeV}$ only for the $52.5 \%$ of events with at least one track associated to a hit on the TOF detector the $t_{\mathrm{ev}}^{\mathrm{TOF}}$ can be provided. In $\mathrm{p}-\mathrm{Pb}$ collisions this fraction increases to $81.8 \%$ reaching $99.6 \%$ in $\mathrm{Pb}-\mathrm{Pb}$ collisions. 

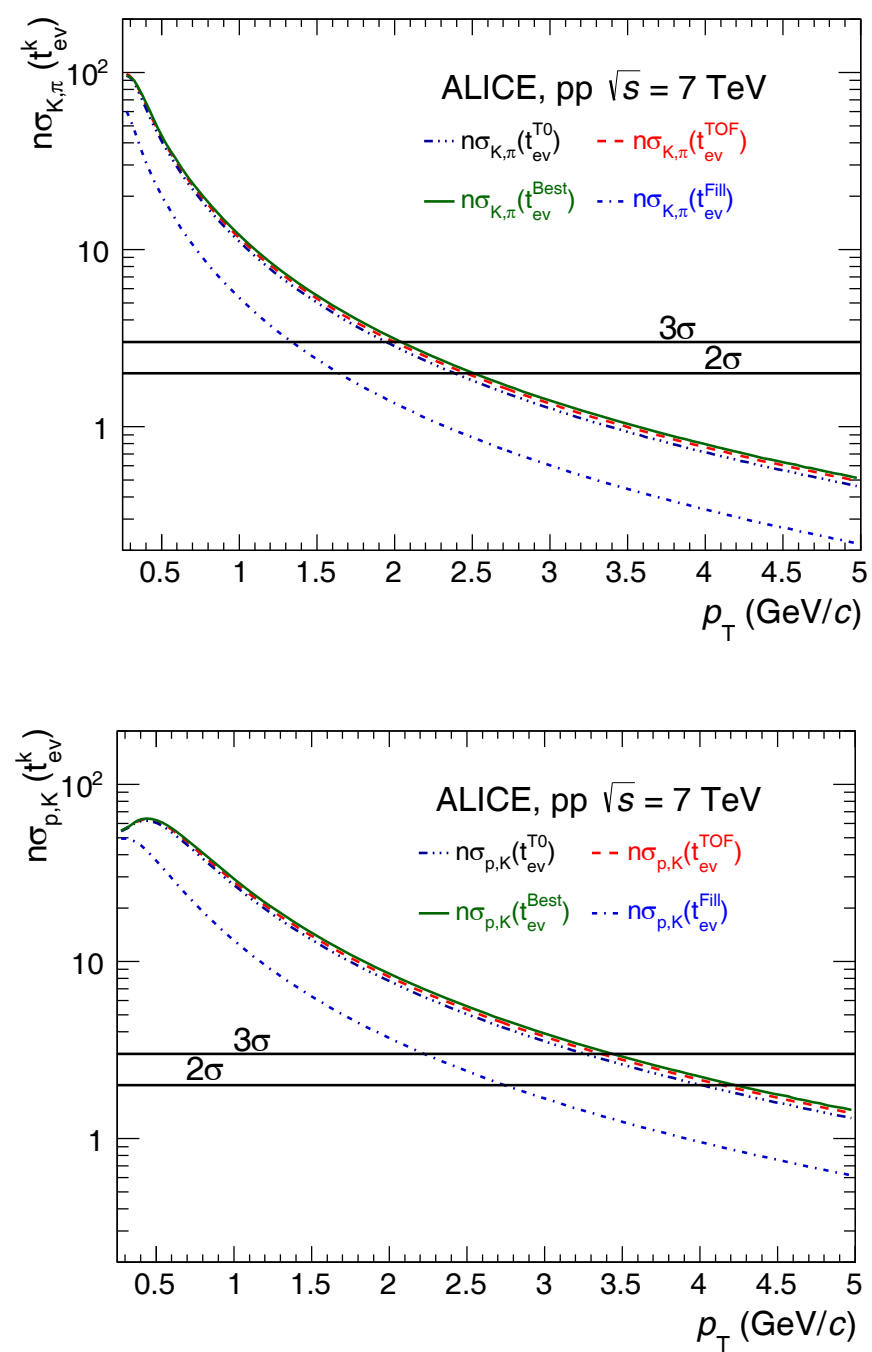

Fig. 9. K- $\pi$ (top) and p-K (bottom) separation power as a function of the transverse momentum of a track when $t_{\mathrm{ev}}^{\mathrm{TOF}}$ (dashed line), $t_{\mathrm{ev}}^{\mathrm{T} 0}$ (dash-dotted line), $t_{\mathrm{ev}}^{\mathrm{Best}}$ (solid line) and $t_{\mathrm{ev}}^{\mathrm{Fill}}$ (dotted line) are used.

To increase the PID performance it is important to use the $t_{\mathrm{ev}}^{\text {Best }}$ which combines the high $t_{\mathrm{ev}}^{\mathrm{T} 0}$ efficiency at low multiplicity events with the better $t_{\mathrm{ev}}^{\mathrm{TOF}}$ resolution at high multiplicity events. Finally, the impact of the method used for the event collision time determination on the TOF PID performance has been discussed, showing how it gets better when $t_{\mathrm{ev}}$ is computed event-by-event improving for example a three sigma $\pi$-K separation from $1.3 \mathrm{GeV} / c$ to $2 \mathrm{GeV} / c$ with respect to when the $t_{\mathrm{ev}}^{\text {Fill }}$ has to be used.

The ALICE Collaboration would like to thank all its engineers and technicians for their invaluable contributions to the construction of the experiment and the CERN accelerator teams for the outstanding performance of the LHC complex. The ALICE Collaboration gratefully acknowledges the resources and support provided by all Grid centres and the Worldwide LHC Computing Grid (WLCG) collaboration. The ALICE Collaboration acknowledges the following funding agencies for their support in building and running the ALICE detector: A. I. Alikhanyan National Science Laboratory (Yerevan Physics Institute) Foundation (ANSL), State Committee of Science and World Federation of Scientists (WFS), Armenia; Austrian Academy of Sciences and Nationalstiftung für Forschung, Technologie und Entwicklung, Austria; Conselho Nacional de Desenvolvimento Científico e Tecnológico (CNPq), Financiadora de Estudos e Projetos (Finep), Fundação de Amparo à Pesquisa do Estado de São Paulo (FAPESP) and, Brazil; Ministry of Education of China (MOEC), Ministry of Science \& Technology of China (MSTC) and National Natural Science Foundation of China (NSFC), China; Ministry of Science, Education and Sport and Croatian Science Foundation, Croatia; Ministry of Education, Youth and Sports of the Czech Republic, Czech Republic; The Danish Council for Independent Research - Natural Sciences, Danish National Research Foundation (DNRF) and The Carlsberg Foundation, Denmark; Helsinki Institute of Physics (HIP), Finland; Institut National de Physique Nucléaire et de Physique des Particules 
(IN2P3) and Centre National de la Recherche Scientifique (CNRS) and Commissariat à l'Energie Atomique (CEA), France; Bundesministerium für Bildung, Wissenschaft, Forschung und Technologie (BMBF) and GSI Helmholtzzentrum für Schwerionenforschung GmbH, Germany; Ministry of Education, Research and Religious Affairs, Greece; National Research, Development and Innovation Office, Hungary; Department of Atomic Energy Government of India (DAE), India; Indonesian Institute of Science, Indonesia; Istituto Nazionale di Fisica Nucleare (INFN) and Centro Fermi - Museo Storico della Fisica e Centro Studi e Ricerche Enrico Fermi, Italy; Japanese Ministry of Education, Culture, Sports, Science and Technology (MEXT), Japan Society for the Promotion of Science (JSPS) KAKENHI and Institute for Innovative Science and Technology, Nagasaki Institute of Applied Science (IIST), Japan; Consejo Nacional de Ciencia (CONACYT) y Tecnología, through Fondo de Cooperación Internacional en Ciencia y Tecnología (FONCICYT) and Dirección General de Asuntos del Personal Academico (DGAPA), Mexico; Nationaal instituut voor subatomaire fysica (Nikhef), Netherlands; The Research Council of Norway, Norway; Commission on Science and Technology for Sustainable Development in the South (COMSATS), Pakistan; Pontificia Universidad Católica del Perú, Peru; Ministry of Science and Higher Education and National Science Centre, Poland; Korea Institute of Science and Technology Information and National Research Foundation of Korea (NRF), Republic of Korea; Romanian National Agency for Science, Technology and Innovation and Ministry of Education and Scientific Research, Institute of Atomic Physics, Romania; Ministry of Education and Science of the Russian Federation, National Research Centre Kurchatov Institute and Joint Institute for Nuclear Research (JINR), Russia; Ministry of Education, Science, Research and Sport of the Slovak Republic, Slovakia; National Research Foundation of South Africa, South Africa; Centro de Investigaciones Energéticas, Medioambientales y Tecnológicas (CIEMAT), Centro de Aplicaciones Tecnológicas y Desarrollo Nuclear (CEADEN), Cubaenergía, Cuba and Ministerio de Ciencia e Innovacion, Spain; Swedish Research Council (VR) and Knut \& Alice Wallenberg Foundation (KAW), Sweden; European Organization for Nuclear Research, Switzerland; Suranaree University of Technology (SUT), National Science and Technology Development Agency (NSDTA) and Office of the Higher Education Commission under NRU project of Thailand, Thailand; Turkish Atomic Energy Agency (TAEK), Turkey; National Academy of Sciences of Ukraine, Ukraine; Science and Technology Facilities Council (STFC), United Kingdom; United States Department of Energy, Office of Nuclear Physics (DOE NP) and National Science Foundation of the United States of America (NSF), United States of America.

\section{Appendix A. The ALICE Collaboration}

J. Adam ${ }^{39}$, D. Adamová86, M.M. Aggarwal ${ }^{90}$, G. Aglieri Rinella ${ }^{35}$, M. Agnello $^{113,31}$, N. Agrawal ${ }^{48}$, Z. Ahammed $^{137}$, S. Ahmad ${ }^{18}$, S.U. Ahn ${ }^{70}$, S. Aiola ${ }^{141}$, A. Akindinov ${ }^{55}$, S.N. Alam ${ }^{137}$, D.S.D. Albuquerque ${ }^{124}$, D. Aleksandrov ${ }^{82}$, B. Alessandro ${ }^{113}$, D. Alexandre ${ }^{104}$, R. Alfaro Molina ${ }^{65}$, A. Alici ${ }^{12,107}$, A. Alkin ${ }^{3}$, J. Alme $^{22,37}$, T. Alt $^{42}$, S. Altinpinar ${ }^{22}$, I. Altsybeev ${ }^{136}$, C. Alves Garcia Prado ${ }^{123}$, M. An ${ }^{7}$, C. Andrei ${ }^{80}$, H.A. Andrews ${ }^{104}$, A. Andronic ${ }^{100}$, V. Anguelov ${ }^{96}$, C. Anson ${ }^{89}$, T. Antičićc ${ }^{101}$, F. Antinori ${ }^{110}$, P. Antonioli ${ }^{107}$, R. Anwar ${ }^{126}$, L. Aphecetche ${ }^{116}$, H. Appelshäuser ${ }^{61}$, S. Arcelli ${ }^{27}$, R. Arnaldi ${ }^{113}$, O.W. Arnold 97,36 , I.C. Arsene ${ }^{21}$, M. Arslandok ${ }^{61}$, B. Audurier ${ }^{116}$, A. Augustinus ${ }^{35}$, R. Averbeck ${ }^{100}$, M.D. Azmi ${ }^{18}$, A. Badalà ${ }^{109}$, Y.W. Baek ${ }^{69}$, S. Bagnasco ${ }^{113}$, R. Bailhache $^{61}$, R. Bala ${ }^{93}$, S. Balasubramanian ${ }^{141}$, A. Baldisseri ${ }^{15}$, R.C. Baral ${ }^{58}$, A.M. Barbano ${ }^{26}$, R. Barbera ${ }^{28}$, F. Barile ${ }^{33}$, G.G. Barnaföldi ${ }^{140}$, L.S. Barnby ${ }^{35,104}$, V. Barret ${ }^{72}$, P. Bartalini ${ }^{7}$, K. Barth ${ }^{35}$, J. Bartke ${ }^{120, c}$, E. Bartsch $^{61}$, M. Basile ${ }^{27}$, N. Bastid ${ }^{72}$, S. Basu ${ }^{137}$, B. Bathen ${ }^{62}$, G. Batigne ${ }^{116}$, A. Batista Camejo ${ }^{72}$, B. Batyunya ${ }^{68}$, P.C. Batzing ${ }^{21}$, I.G. Bearden ${ }^{83}$, H. Beck ${ }^{96}$, C. Bedda ${ }^{31}$, N.K. Behera ${ }^{51}$, I. Belikov ${ }^{66}$, F. Bellini ${ }^{27}$, H. Bello Martinez ${ }^{2}$, R. Bellwied ${ }^{126}$, L.G.E. Beltran ${ }^{122}$, V. Belyaev ${ }^{77}$, G. Bencedi ${ }^{140}$, S. Beole ${ }^{26}$, A. Bercuci ${ }^{80}$, Y. Berdnikov ${ }^{88}$, D. Berenyi ${ }^{140}$, R.A. Bertens ${ }^{129,54}$, D. Berzano ${ }^{35}$, L. Betev ${ }^{35}$, A. Bhasin ${ }^{93}$, I.R. Bhat ${ }^{93}$, A.K. Bhati ${ }^{90}$, B. Bhattacharjee ${ }^{44}$, J. Bhom ${ }^{120}$, L. Bianchi ${ }^{126}$, N. Bianchi ${ }^{74}$, C. Bianchin ${ }^{139}$, J. Bielčík ${ }^{39}$, J. Bielčíková ${ }^{86}$, A. Bilandzic ${ }^{36,97}$, G. Biro ${ }^{140}$, R. Biswas ${ }^{4}$, S. Biswas ${ }^{81,4}$, S. Bjelogrlic ${ }^{54}$, J.T. Blair ${ }^{121}$, D. Blau ${ }^{82}$, C. Blume ${ }^{61}$, F. Bock ${ }^{76,96}$, A. Bogdanov ${ }^{77}$, L. Boldizsár ${ }^{140}$, M. Bombara ${ }^{40}$, M. Bonora ${ }^{35}$, J. Book ${ }^{61}$, H. Borel ${ }^{15}$, A. Borissov $^{99}$, M. Borri ${ }^{128}$, E. Botta ${ }^{26}$, C. Bourjau ${ }^{83}$, P. Braun-Munzinger ${ }^{100}$, M. Bregant ${ }^{123}$, T.A. Broker ${ }^{61}$, T.A. Browning ${ }^{98}$,' M. Broz ${ }^{39}$, E.J. Brucken ${ }^{46}$, E. Bruna ${ }^{113}$, G.E. Bruno ${ }^{33}$, D. Budnikov ${ }^{102}$, H. Buesching ${ }^{61}$, S. Bufalino ${ }^{31,26}$, P. Buhler ${ }^{115}$, S.A.I. Buitron ${ }^{63}$, P. Buncic ${ }^{35}$, O. Busch ${ }^{132}$, Z. Buthelezi ${ }^{67}$, J.B. Butt ${ }^{16}$, J.T. Buxton ${ }^{19}$, J. Cabala ${ }^{118}$, D. Caffarri ${ }^{35}$, H. Caines ${ }^{141}$, A. Caliva ${ }^{54}$, E. Calvo Villar ${ }^{105}$, P. Camerini ${ }^{25}$, F. Carena ${ }^{35}$, W. Carena ${ }^{35}$,

F. Carnesecchi ${ }^{12,27}$, J. Castillo Castellanos ${ }^{15}$, A.J. Castro ${ }^{129}$, E.A.R. Casula ${ }^{24}$, C. Ceballos Sanchez ${ }^{9}$, J. Cepila ${ }^{39}$, P. Cerello ${ }^{113}$, J. Cerkala ${ }^{118}$, B. Chang ${ }^{127}$, S. Chapeland ${ }^{35}$, M. Chartier ${ }^{128}$, J.L. Charvet ${ }^{15}$, S. Chattopadhyay ${ }^{137}$, S. Chattopadhyay ${ }^{103}$, A. Chauvin ${ }^{97,36}$, V. Chelnokov ${ }^{3}$, M. Cherney ${ }^{89}$, C. Cheshkov ${ }^{134}$, B. Cheynis ${ }^{134}$,

V. Chibante Barroso ${ }^{35}$, D.D. Chinellato ${ }^{124}$, S. Cho ${ }^{51}$, P. Chochula ${ }^{35}$, K. Choi ${ }^{99}$, M. Chojnacki ${ }^{83}$, S. Choudhury ${ }^{137}$, P. Christakoglou ${ }^{84}$, C.H. Christensen ${ }^{83}$, P. Christiansen ${ }^{34}$, T. Chujo ${ }^{132}$, S.U. Chung ${ }^{99}$, C. Cicalo ${ }^{108}$, L. Cifarelli ${ }^{12,27}$, F. Cindolo ${ }^{107}$, J. Cleymans ${ }^{92}$, F. Colamaria ${ }^{33}$, D. Colella ${ }^{56,35}$, A. Collu ${ }^{76}$, M. Colocci ${ }^{27}$, G. Conesa Balbastre ${ }^{73}$, Z. Conesa del Valle ${ }^{52}$, M.E. Connors ${ }^{141, d}$, J.G. Contreras ${ }^{39}$, T.M. Cormier ${ }^{87}$, Y. Corrales Morales ${ }^{113}$,

I. Cortés Maldonado ${ }^{2}$, P. Cortese ${ }^{32}$, M.R. Cosentino ${ }^{123,125}$, F. Costa ${ }^{35}$, J. Crkovská $^{52}$, P. Crochet ${ }^{72}$,

R. Cruz Albino ${ }^{11}$, E. Cuautle ${ }^{63}$, L. Cunqueiro ${ }^{35,62}$, T. Dahms ${ }^{36,97}$, A. Dainese ${ }^{110}$, M.C. Danisch ${ }^{96}$, A. Danu $^{59}$, D. $\operatorname{Das}^{103}$, I. Das ${ }^{103}$, S. Das ${ }^{4}$, A. $\operatorname{Dash}^{81}$, S. Dash ${ }^{48}$, S. De ${ }^{49,123}$, A. De Caro ${ }^{30}$, G. de Cataldo ${ }^{106}$, C. de Conti ${ }^{123}$,

\footnotetext{
c Deceased.

d Also at: Georgia State University, Atlanta, Georgia, USA.
} 
J. de Cuveland ${ }^{42}$, A. De Falco ${ }^{24}$, D. De Gruttola ${ }^{30,12}$, N. De Marco ${ }^{113}$, S. De Pasquale ${ }^{30}$, R.D. De Souza ${ }^{124}$, A. Deisting ${ }^{100,96}$, A. Deloff ${ }^{79}$, C. Deplano ${ }^{84}$, P. Dhankher ${ }^{48}$, D. Di Bari ${ }^{33}$, A. Di Mauro ${ }^{35}$, P. Di Nezza $^{74}$, B. Di Ruzza ${ }^{110}$, M.A. Diaz Corchero ${ }^{10}$, T. Dietel ${ }^{92}$, P. Dillenseger ${ }^{61}$, R. Divià ${ }^{35}$, Ø. Djuvsland ${ }^{22}$, A. Dobrin $^{84,35}$, D. Domenicis Gimenez ${ }^{123}$, B. Dönigus ${ }^{61}$, O. Dordic ${ }^{21}$, T. Drozhzhova ${ }^{61}$, A.K. Dubey ${ }^{137}$, A. Dubla ${ }^{100}$, L. Ducroux ${ }^{134}$ A.K. Duggal ${ }^{90}$, P. Dupieux ${ }^{72}$, R.J. Ehlers ${ }^{141}$, D. Elia ${ }^{106}$, E. Endress ${ }^{105}$, H. Engel ${ }^{60}$, E. Epple ${ }^{141}$, B. Erazmus ${ }^{116}$, F. Erhardt ${ }^{133}$, B. Espagnon ${ }^{52}$, S. Esumi ${ }^{132}$, G. Eulisse ${ }^{35}$, J. Eum ${ }^{99}$, D. Evans ${ }^{104}$, S. Evdokimov ${ }^{114}$, G. Eyyubova ${ }^{39}$, L. Fabbietti ${ }^{36,97}$, D. Fabris ${ }^{110}$, J. Faivre ${ }^{73}$, A. Fantoni ${ }^{74}$, M. Fasel ${ }^{87,76}$, L. Feldkamp ${ }^{62}$, A. Feliciello ${ }^{113}$, G. Feofilov ${ }^{136}$ J. Ferencei ${ }^{86}$, A. Fernández Téllez ${ }^{2}$, E.G. Ferreiro ${ }^{17}$, A. Ferretti ${ }^{26}$, A. Festanti ${ }^{29}$, V.J.G. Feuillard ${ }^{72,15}$, J. Figiel ${ }^{120}$, M.A.S. Figueredo ${ }^{123}$, S. Filchagin ${ }^{102}$, D. Finogeev ${ }^{53}$, F.M. Fionda ${ }^{24}$, E.M. Fiore ${ }^{33}$, M. Floris ${ }^{35}$, S. Foertsch ${ }^{67}$, P. Foka ${ }^{100}$, S. Fokin ${ }^{82}$, E. Fragiacomo ${ }^{112}$, A. Francescon ${ }^{35}$, A. Francisco ${ }^{116}$, U. Frankenfeld ${ }^{100}$, G.G. Fronze ${ }^{26}$, U. Fuchs ${ }^{35}$, C. Furget ${ }^{73}$, A. Furs ${ }^{53}$, M. Fusco Girard ${ }^{30}$, J.J. Gaardhøje ${ }^{83}$, M. Gagliardi ${ }^{26}$, A.M. Gago ${ }^{105}$,

K. Gajdosova ${ }^{83}$, M. Gallio ${ }^{26}$, C.D. Galvan ${ }^{122}$, D.R. Gangadharan ${ }^{76}$, P. Ganoti ${ }^{91,35}$, C. Gao ${ }^{7}$, C. Garabatos ${ }^{100}$, E. Garcia-Solis ${ }^{13}$, K. Garg ${ }^{28}$, P. Garg ${ }^{49}$, C. Gargiulo ${ }^{35}$, P. Gasik ${ }^{97,36}$, E.F. Gauger ${ }^{121}$, M.B. Gay Ducati ${ }^{64}$, M. Germain ${ }^{116}$, P. Ghosh ${ }^{137}$, S.K. Ghosh ${ }^{4}$, P. Gianotti ${ }^{74}$, P. Giubellino ${ }^{113,35}$, P. Giubilato ${ }^{29}$, E. Gladysz-Dziadus $^{120}$, P. Glässel ${ }^{96}$, D.M. Goméz Coral ${ }^{65}$, A. Gomez Ramirez ${ }^{60}$, A.S. Gonzalez ${ }^{35}$, V. Gonzalez ${ }^{10}$, P. González-Zamora ${ }^{10}$, S. Gorbunov ${ }^{42}$, L. Görlich ${ }^{120}$, S. Gotovac ${ }^{119}$, V. Grabski ${ }^{65}$, L.K. Graczykowski ${ }^{138}$, K.L. Graham ${ }^{104}$, L. Greiner ${ }^{76}$, A. Grelli ${ }^{54}$, C. Grigoras ${ }^{35}$, V. Grigoriev ${ }^{77}$, A. Grigoryan ${ }^{1}$, S. Grigoryan ${ }^{68}$, N. Grion ${ }^{112}$, J.M. Gronefeld ${ }^{100}$, J.F. Grosse-Oetringhaus ${ }^{35}$, R. Grosso ${ }^{100}$, L. Gruber ${ }^{115}$, F. Guber ${ }^{53}$, R. Guernane ${ }^{73,35}$, B. Guerzoni ${ }^{27}$, K. Gulbrandsen ${ }^{83}$, T. Gunji ${ }^{131}$, A. Gupta ${ }^{93}$, R. Gupta ${ }^{93}$, I.B. Guzman ${ }^{2}$, R. Haake ${ }^{35,62}$, C. Hadjidakis ${ }^{52}$, H. Hamagaki ${ }^{131,78}$, G. Hamar ${ }^{140}$, J.C. Hamon ${ }^{66}$, J.W. Harris ${ }^{141}$, A. Harton ${ }^{13}$, D. Hatzifotiadou ${ }^{107}$, S. Hayashi ${ }^{131}$, S.T. Heckel ${ }^{61}$, E. Hellbär ${ }^{61}$, H. Helstrup ${ }^{37}$, A. Herghelegiu ${ }^{80}$, G. Herrera Corral ${ }^{11}$, F. Herrmann ${ }^{62}$, B.A. Hess ${ }^{95}$, K.F. Hetland ${ }^{37}$, H. Hillemanns ${ }^{35}$, B. Hippolyte ${ }^{66}$, J. Hladky ${ }^{57}$, D. Horak ${ }^{39}$, R. Hosokawa ${ }^{132}$, P. Hristov ${ }^{35}$, C. Hughes ${ }^{129}$, T.J. Humanic ${ }^{19}$, N. Hussain ${ }^{44}$, T. Hussain ${ }^{18}$, D. Hutter ${ }^{42}$, D.S. Hwang ${ }^{20}$, R. Ilkaev ${ }^{102}$, M. Inaba ${ }^{132}$, M. Ippolitov ${ }^{82,77}$, M. Irfan ${ }^{18}$, V. Isakov ${ }^{53}$, M.S. Islam ${ }^{49}$, M. Ivanov ${ }^{35,100}$, V. Ivanov ${ }^{88}$, V. Izucheev ${ }^{114}$, B. Jacak $^{76}$, N. Jacazio ${ }^{27}$, P.M. Jacobs ${ }^{76}$, M.B. Jadhav ${ }^{48}$, S. Jadlovska ${ }^{118}$, J. Jadlovsky ${ }^{118}$, C. Jahnke ${ }^{123,36}$, M.J. Jakubowska ${ }^{138}$, M.A. Janik ${ }^{138}$, P.H.S.Y. Jayarathna ${ }^{126}$, C. Jena ${ }^{81}$, S. Jena ${ }^{126}$, R.T. Jimenez Bustamante ${ }^{100}$, P.G. Jones $^{104}$,

A. Jusko ${ }^{104}$, P. Kalinak ${ }^{56}$, A. Kalweit ${ }^{35}$, J.H. Kang ${ }^{142}$, V. Kaplin ${ }^{77}$, S. Kar ${ }^{137}$, A. Karasu Uysal ${ }^{71}$, O. Karavichev ${ }^{53}$, T. Karavicheva ${ }^{53}$, L. Karayan ${ }^{100,96}$, E. Karpechev ${ }^{53}$, U. Kebschull ${ }^{60}$, R. Keidel ${ }^{143}$, D.L.D. Keijdener ${ }^{54}$, M. Keil ${ }^{35}$, M. Mohisin Khan ${ }^{18, e}$, P. Khan ${ }^{103}$, S.A. Khan ${ }^{137}$, A. Khanzadeev ${ }^{88}$, Y. Kharlov ${ }^{114}$, A. Khatun ${ }^{18}$, A. Khuntia ${ }^{49}$, B. Kileng ${ }^{37}$, D.W. Kim ${ }^{43}$, D.J. Kim ${ }^{127}$, D. Kim ${ }^{142}$, H. Kim ${ }^{142}$, J.S. Kim ${ }^{43}$, J. Kim ${ }^{96}$, M. Kim $^{51}$, M. Kim$^{142}$, S. Kim ${ }^{20}$, T. Kim ${ }^{142}$, S. Kirsch ${ }^{42}$, I. Kisel ${ }^{42}$, S. Kiselev ${ }^{55}$, A. Kisiel ${ }^{138}$, G. Kiss ${ }^{140}$, J.L. Klay ${ }^{6}$, C. Klein ${ }^{61}$, J. Klein ${ }^{35}$, C. Klein-Bösing ${ }^{62}$, S. Klewin ${ }^{96}$, A. Kluge ${ }^{35}$, M.L. Knichel ${ }^{96}$, A.G. Knospe ${ }^{121,126}$, C. Kobdaj117, M. Kofarago ${ }^{35}$, T. Kollegger ${ }^{100}$, A. Kolojvari ${ }^{136}$, V. Kondratiev ${ }^{136}$, N. Kondratyeva ${ }^{77}$, E. Kondratyuk ${ }^{114}$, A. Konevskikh ${ }^{53}$, M. Kopcik ${ }^{118}$, M. Kour ${ }^{93}$, C. Kouzinopoulos ${ }^{35}$, O. Kovalenko ${ }^{79}$, V. Kovalenko ${ }^{136}$, M. Kowalski ${ }^{120}$,

G. Koyithatta Meethaleveedu ${ }^{48}$, I. Králik ${ }^{56}$, A. Kravčáková ${ }^{40}$, M. Krivda ${ }^{104,56}$, F. Krizek ${ }^{86}$, E. Kryshen ${ }^{88,35}$, M. Krzewicki ${ }^{42}$, A.M. Kubera ${ }^{19}$, V. Kučera ${ }^{86}$, C. Kuhn ${ }^{66}$, P.G. Kuijer ${ }^{84}$, A. Kumar ${ }^{93}$, J. Kumar ${ }^{48}$, L. Kumar ${ }^{90}$, S. Kumar ${ }^{48}$, S. Kundu ${ }^{81}$, P. Kurashvili ${ }^{79}$, A. Kurepin ${ }^{53}$, A.B. Kurepin ${ }^{53}$, A. Kuryakin ${ }^{102}$, S. Kushpil ${ }^{86}$, M.J. Kweon ${ }^{51}$, Y. Kwon ${ }^{142}$, S.L. La Pointe ${ }^{42}$, P. La Rocca ${ }^{28}$, C. Lagana Fernandes ${ }^{123}$, I. Lakomov ${ }^{35}$, R. Langoy ${ }^{41}$, K. Lapidus ${ }^{36,141}$, C. Lara $^{60}$, A. Lardeux ${ }^{15}$, A. Lattuca ${ }^{26}$, E. Laudi ${ }^{35}$, L. Lazaridis ${ }^{35}$, R. Lea ${ }^{25}$, L. Leardini ${ }^{96}$, S. Lee ${ }^{142}$, F. Lehas $^{84}$, S. Lehner ${ }^{115}$, J. Lehrbach ${ }^{42}$, R.C. Lemmon ${ }^{85}$, V. Lenti ${ }^{106}$, E. Leogrande ${ }^{54}$, I. León Monzón ${ }^{122}$, P. Lévai ${ }^{140}$, S. Li ${ }^{7}$, X. Li ${ }^{14}$, J. Lien ${ }^{41}$, R. Lietava ${ }^{104}$, S. Lindal ${ }^{21}$, V. Lindenstruth ${ }^{42}$, C. Lippmann ${ }^{100}$, M.A. Lisa ${ }^{19}$, H.M. Ljunggren ${ }^{34}$, W. Llope ${ }^{139}$, D.F. Lodato ${ }^{54}$, P.I. Loenne ${ }^{22}$, V. Loginov ${ }^{77}$, C. Loizides ${ }^{76}$, X. Lopez $^{72}$, E. López Torres ${ }^{9}$, A. Lowe ${ }^{140}$, P. Luettig ${ }^{61}$, M. Lunardon ${ }^{29}$, G. Luparello ${ }^{25}$, M. Lupi ${ }^{35}$, T.H. Lutz ${ }^{141}$, A. Maevskaya ${ }^{53}$, M. Mager ${ }^{35}$, S. Mahajan ${ }^{93}$, S.M. Mahmood ${ }^{21}$, A. Maire ${ }^{66}$, R.D. Majka ${ }^{141}$, M. Malaev ${ }^{88}$ I. Maldonado Cervantes ${ }^{63}$, L. Malinina ${ }^{68, f}$, D. Mal'Kevich ${ }^{55}$, P. Malzacher ${ }^{100}$, A. Mamonov ${ }^{102}$, V. Manko' F. Manso ${ }^{72}$, V. Manzari ${ }^{106}$, Y. Mao ${ }^{7}$, M. Marchisone ${ }^{67,130}$, J. Mareš ${ }^{57}$, G.V. Margagliotti ${ }^{25}$, A. Margotti ${ }^{107}$ ', J. Margutti ${ }^{54}$, A. Marín ${ }^{100}$, C. Markert ${ }^{121}$, M. Marquard ${ }^{61}$, N.A. Martin ${ }^{100}$, P. Martinengo ${ }^{35}$, M.I. Martínez $^{2}$, G. Martínez García ${ }^{116}$, M. Martinez Pedreira ${ }^{35}$, A. Mas ${ }^{123}$, S. Masciocchi ${ }^{100}$, M. Masera ${ }^{26}$, A. Masoni ${ }^{108}$, A. Mastroserio ${ }^{33}$, A. Matyja ${ }^{120,129}$, C. Mayer ${ }^{120}$, J. Mazer ${ }^{129}$, M. Mazzilli ${ }^{33}$, M.A. Mazzoni ${ }^{111}$, F. Meddi ${ }^{23}$, Y. Melikyan ${ }^{77}$, A. Menchaca-Rocha ${ }^{65}$, E. Meninno ${ }^{30}$, J. Mercado Pérez ${ }^{96}$, M. Meres ${ }^{38}$, S. Mhlanga ${ }^{92}$, Y. Miake $^{132}$, M.M. Mieskolainen ${ }^{46}$, K. Mikhaylov ${ }^{55,68}$, L. Milano ${ }^{76}$, J. Milosevic ${ }^{21}$, A. Mischke ${ }^{54}$, A.N. Mishra ${ }^{49}$, T. Mishra $^{58}$, D. Miśkowiec ${ }^{100}$, J. Mitra ${ }^{137}$, C.M. Mitu ${ }^{59}$, N. Mohammadi ${ }^{54}$, B. Mohanty ${ }^{81}$, L. Molnar ${ }^{116}$, E. Montes ${ }^{10}$, D.A. Moreira De Godoy ${ }^{62}$, L.A.P. Moreno ${ }^{2}$, S. Moretto ${ }^{29}$, A. Morreale ${ }^{116}$, A. Morsch ${ }^{35}$, V. Muccifora ${ }^{74}$, E. Mudnic ${ }^{119}$, D. Mühlheim ${ }^{62}$, S. Muhuri ${ }^{137}$, M. Mukherjee ${ }^{137}$, J.D. Mulligan ${ }^{141}$, M.G. Munhoz ${ }^{123}$, K. Münning $^{45}$, R.H. Munzer ${ }^{97,61,36}$, H. Murakami ${ }^{131}$, S. Murray ${ }^{67}$, L. Musa ${ }^{35}$, J. Musinsky ${ }^{56}$, C.J. Myers ${ }^{126}$, B. Naik ${ }^{48}$, R. Nair ${ }^{79}$, B.K. Nandi ${ }^{48}$, R. Nania ${ }^{107}$, E. Nappi ${ }^{106}$, M.U. Naru ${ }^{16}$, H. Natal da Luz ${ }^{123}$, C. Nattrass ${ }^{129}$, S.R. Navarro ${ }^{2}$, K. Nayak ${ }^{81}$, R. Nayak ${ }^{48}$, T.K. Nayak ${ }^{137}$, S. Nazarenko ${ }^{102}$, A. Nedosekin ${ }^{55}$, R.A. Negrao De Oliveira ${ }^{35}$, L. Nellen ${ }^{63}$,

e Also at: Department of Applied Physics, Aligarh Muslim University, Aligarh, India.

f Also at: M.V. Lomonosov Moscow State University, D.V. Skobeltsyn Institute of Nuclear, Physics, Moscow, Russia. 


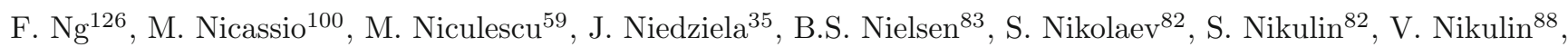

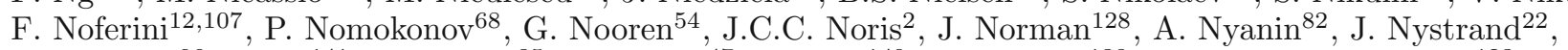
H. Oeschler ${ }^{96}$, S. Oh ${ }^{141}$, A. Ohlson ${ }^{35}$, T. Okubo ${ }^{47}$, L. Olah ${ }^{140}$, J. Oleniacz ${ }^{138}$, A.C. Oliveira Da Silva ${ }^{123}$, M.H. Oliver ${ }^{141}$, J. Onderwaater ${ }^{100}$, C. Oppedisano ${ }^{113}$, R. Orava ${ }^{46}$, M. Oravec ${ }^{118}$, A. Ortiz Velasquez ${ }^{63}$, A. Oskarsson ${ }^{34}$, J. Otwinowski ${ }^{120}$,K. Oyama ${ }^{78}$, M. Ozdemir ${ }^{61}$, Y. Pachmayer ${ }^{96}$, V. Pacik ${ }^{83}$, D. Pagano ${ }^{135,26}$, P. Pagano ${ }^{30}$, G. Paić ${ }^{63}$, S.K. Pal ${ }^{137}$, P. Palni ${ }^{7}$, J. Pan ${ }^{139}$, A.K. Pandey ${ }^{48}$, V. Papikyan ${ }^{1}$, G.S. Pappalardo ${ }^{109}$, P. Pareek ${ }^{49}$, J. Park ${ }^{51}$, W.J. Park ${ }^{100}$, S. Parmar ${ }^{90}$, A. Passfeld ${ }^{62}$, V. Paticchio ${ }^{106}$, R.N. Patra ${ }^{137}$, B. Paul ${ }^{113}$, H. Pei ${ }^{7}$, T. Peitzmann ${ }^{54}$, X. Peng 7 , H. Pereira Da Costa ${ }^{15}$, D. Peresunko ${ }^{77,82}$, E. Perez Lezama ${ }^{61}$, V. Peskov ${ }^{61}$, Y. Pestov ${ }^{5}$, V. Petráček ${ }^{39}$, V. Petrov ${ }^{114}$, M. Petrovici ${ }^{80}$, C. Petta ${ }^{28}$, S. Piano ${ }^{112}$, M. Pikna ${ }^{38}$, P. Pillot ${ }^{116}$, L.O.D.L. Pimentel ${ }^{83}$, O. Pinazza ${ }^{35,107}$, L. Pinsky ${ }^{126}$, D.B. Piyarathna ${ }^{126}$, M. Płoskoń ${ }^{76}$, M. Planinic ${ }^{133}$, J. Pluta ${ }^{138}$, S. Pochybova ${ }^{140}$, P.L.M. Podesta-Lerma ${ }^{122}$, M.G. Poghosyan ${ }^{87}$, B. Polichtchouk ${ }^{114}$, N. Poljak ${ }^{133}$, W. Poonsawat ${ }^{117}$, A. Pop ${ }^{80}$, H. Poppenborg ${ }^{62}$, S. Porteboeuf-Houssais ${ }^{72}$, J. Porter ${ }^{76}$, J. Pospisil ${ }^{86}$, V. Pozdniakov ${ }^{68}$, S.K. Prasad ${ }^{4}$, R. Preghenella ${ }^{107,35}$, F. Prino ${ }^{113}$, C.A. Pruneau ${ }^{139}$, I. Pshenichnov ${ }^{53}$, M. Puccio ${ }^{26}$, G. Puddu ${ }^{24}$, P. Pujahari ${ }^{139}$, V. Punin ${ }^{102}$, J. Putschke ${ }^{139}$, H. Qvigstad ${ }^{21}$, A. Rachevski ${ }^{112}$, S. Raha ${ }^{4}$, S. Rajput ${ }^{93}$, J. Rak ${ }^{127}$,

A. Rakotozafindrabe ${ }^{15}$, L. Ramello ${ }^{32}$, F. Rami ${ }^{66}$, D.B. Rana ${ }^{126}$, R. Raniwala ${ }^{94}$, S. Raniwala ${ }^{94}$, S.S. Räsänen ${ }^{46}$, B.T. Rascanu ${ }^{61}$, D. Rathee ${ }^{90}$, V. Ratza ${ }^{45}$, I. Ravasenga ${ }^{26}$, K.F. Read ${ }^{87,129}$, K. Redlich ${ }^{79}$, A. Rehman ${ }^{22}$,

P. Reichelt ${ }^{61}$, F. Reidt ${ }^{35,96}$, X. Ren ${ }^{7}$, R. Renfordt ${ }^{61}$, A.R. Reolon ${ }^{74}$, A. Reshetin ${ }^{53}$, K. Reygers ${ }^{96}$, V. Riabov ${ }^{88}$, R.A. Ricci ${ }^{75}$, T. Richert ${ }^{34,54}$, M. Richter ${ }^{21}$, P. Riedler ${ }^{35}$, W. Riegler ${ }^{35}$, F. Riggi ${ }^{28}$, C. Ristea ${ }^{59}$,

M. Rodríguez Cahuantzi ${ }^{2}$, K. Røed ${ }^{21}$, E. Rogochaya ${ }^{68}$, D. Rohr ${ }^{2}$, D. Röhrich ${ }^{22}$, F. Ronchetti ${ }^{74,35}$, L. Ronflette ${ }^{116}$, P. Rosnet ${ }^{72}$, A. Rossi ${ }^{29}$, F. Roukoutakis ${ }^{91}$, A. Roy ${ }^{49}$, C. Roy ${ }^{66}$, P. Roy ${ }^{103}$, A.J. Rubio Montero ${ }^{10}$, R. Rui ${ }^{25}$,

R. Russo ${ }^{26}$, E. Ryabinkin ${ }^{82}$, Y. Ryabov ${ }^{88}$, A. Rybicki ${ }^{120}$, S. Saarinen ${ }^{46}$, S. Sadhu ${ }^{137}$, S. Sadovsky ${ }^{114}$, K. Šafař́k ${ }^{35}$, B. Sahlmuller61, B. Sahoo ${ }^{48}$, P. Sahoo ${ }^{49}$, R. Sahoo ${ }^{49}$, S. Sahoo ${ }^{58}$, P.K. Sahu ${ }^{58}$, J. Saini ${ }^{137}$, S. Sakai ${ }^{132,74}$, M.A. Saleh ${ }^{139}$, J. Salzwedel ${ }^{19}$, S. Sambyal ${ }^{93}$, V. Samsonov ${ }^{77,88}$, A. Sandoval ${ }^{65}$, M. Sano ${ }^{132}$, D. Sarkar ${ }^{137}$, N. Sarkar ${ }^{137}$, P. Sarma ${ }^{44}$, M.H.P. Sas ${ }^{54}$, E. Scapparone ${ }^{107}$, F. Scarlassara ${ }^{29}$, R.P. Scharenberg 98 , C. Schiaua ${ }^{80}$, R. Schicker ${ }^{96}$, C. Schmidt ${ }^{100}$, H.R. Schmidt ${ }^{95}$, M. Schmidt ${ }^{95}$, J. Schukraft ${ }^{35}$, Y. Schutz ${ }^{116,66,35}$, K. Schwarz ${ }^{100}$, K. Schweda ${ }^{100}$, G. Scioli ${ }^{27}$, E. Scomparin ${ }^{113}$, R. Scott ${ }^{129}$, M. Ś. Sefćík ${ }^{40}$, J.E. Seger ${ }^{89}$, Y. Sekiguchi ${ }^{131}$, D. Sekihata ${ }^{47}$, I. Selyuzhenkov ${ }^{100}$, K. Senosi ${ }^{67}$, S. Senyukov ${ }^{3,35}$, E. Serradilla ${ }^{10,65}$, P. Sett ${ }^{48}$, A. Sevcenco ${ }^{59}$, A. Shabanov ${ }^{53}$, A. Shabetai ${ }^{116}$, O. Shadura ${ }^{3}$, R. Shahoyan ${ }^{35}$, A. Shangaraev ${ }^{114}$, A. Sharma ${ }^{93}$, A. Sharma ${ }^{90}$, M. Sharma ${ }^{93}$, M. Sharma ${ }^{33}$, N. Sharma ${ }^{90,129}$, A.I. Sheikh ${ }^{137}$, K. Shigaki ${ }^{47}$, Q. Shou ${ }^{7}$, K. Shtejer ${ }^{9,26}$, Y. Sibiriak ${ }^{82}$, S. Siddhanta ${ }^{108}$, K.M. Sielewicz ${ }^{35}$, T. Siemiarczuk ${ }^{79}$, D. Silvermyr ${ }^{34}$, C. Silvestre ${ }^{73}$, G. Simatovic ${ }^{133}$, G. Simonetti ${ }^{35}$, R. Singaraju ${ }^{137}$, R. Singh ${ }^{81}$, V. Singhal ${ }^{137}$, T. Sinha ${ }^{103}$, B. Sitar ${ }^{38}$, M. Sitta ${ }^{32}$, T.B. Skaali ${ }^{21}$, M. Slupecki ${ }^{127}$, N. Smirnov ${ }^{141}$, R.J.M. Snellings ${ }^{54}$, T.W. Snellman ${ }^{127}$, J. Song ${ }^{99}$, M. Song ${ }^{142}$, Z. Song ${ }^{7}$, F. Soramel ${ }^{29}$, S. Sorensen ${ }^{129}$, F. Sozzi ${ }^{100}$, E. Spiriti ${ }^{74}$, I. Sputowska ${ }^{120}$, B.K. Srivastava ${ }^{98}$, J. Stachel ${ }^{96}$, I. Stan ${ }^{59}$, P. Stankus ${ }^{87}$, E. Stenlund ${ }^{34}$, G. Steyn ${ }^{67}$, J.H. Stiller ${ }^{96}$, D. Stocco ${ }^{116}$, P. Strmen ${ }^{38}$, A.A.P. Suaide ${ }^{123}$, T. Sugitate ${ }^{47}$, C. Suire ${ }^{52}$, M. Suleymanov ${ }^{16}$, M. Suljic ${ }^{25}$, R. Sultanov ${ }^{55}$, M. Šumbera ${ }^{86}$, S. Sumowidagdo ${ }^{50}$, K. Suzuki ${ }^{115}$, S. Swain ${ }^{58}$, A. Szabo ${ }^{38}$, I. Szarka ${ }^{38}$,

A. Szczepankiewicz ${ }^{138}$, M. Szymanski ${ }^{138}$, U. Tabassam ${ }^{16}$, J. Takahashi ${ }^{124}$, G.J. Tambave ${ }^{22}$, N. Tanaka ${ }^{132}$, M. Tarhinis ${ }^{52}$ M. Tariq ${ }^{18}$, M.G. Tarzila ${ }^{80}$, A. Tauro ${ }^{35}$, G. Tejeda Muñoz ${ }^{2}$, A. Telesca ${ }^{35}$, K. Terasaki ${ }^{131}$, C. Terrevoli ${ }^{29}$, B. Teyssier ${ }^{134}$, D. Thakur ${ }^{49}$, D. Thomas ${ }^{121}$, R. Tieulent ${ }^{134}$, A. Tikhonov ${ }^{53}$, A.R. Timmins ${ }^{126}$, A. Toia ${ }^{61}$, S. Tripathy ${ }^{49}$, S. Trogolo ${ }^{26}$, G. Trombetta ${ }^{33}$, V. Trubnikov ${ }^{3}$, W.H. Trzaska ${ }^{127}$, T. Tsuji 131, A. Tumkin ${ }^{102}$, R. Turrisi ${ }^{110}$, T.S. Tveter ${ }^{21}$, K. Ullaland ${ }^{22}$, E.N. Umaka ${ }^{126}$, A. Uras ${ }^{134}$, G.L. Usai ${ }^{24}$, A. Utrobicic ${ }^{133}$, M. Vala ${ }^{56}$, J. Van Der Maarel ${ }^{54}$, J.W. Van Hoorne ${ }^{35}$, M. van Leeuwen ${ }^{54}$, T. Vanat ${ }^{86}$, P. Vande Vyvre ${ }^{35}$, D. Varga ${ }^{140}$,

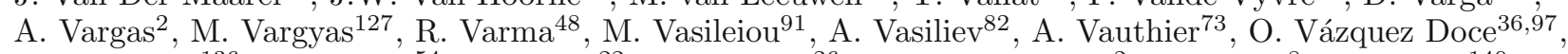
V. Vechernin ${ }^{136}$, A.M. Veen ${ }^{54}$, A. Velure ${ }^{22}$, E. Vercellin ${ }^{26}$, S. Vergara Limón ${ }^{2}$, R. Vernet ${ }^{8}$, R. Vértesi ${ }^{140}$, L. Vickovic ${ }^{119}, S_{\text {S. Vigolo }}^{54}$, J. Viinikainen ${ }^{127}$, Z. Vilakazi ${ }^{130}$, O. Villalobos Baillie ${ }^{104}$, A. Villatoro Tello ${ }^{2}$,

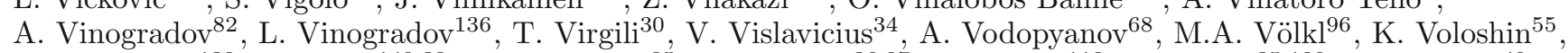
S.A. Voloshin ${ }^{139}$, G. Volpe ${ }^{40,33}$, B. von Haller ${ }^{35}$, I. Vorobyev ${ }^{36,97}$, D. Voscek ${ }^{118}$, D. Vranic ${ }^{35,100}$, J. Vrlákováa ${ }^{40}$, B. Wagner ${ }^{22}$, J. Wagner ${ }^{100}$, H. Wang ${ }^{54}$, M. Wang ${ }^{7}$, D. Watanabe ${ }^{132}$, Y. Watanabe ${ }^{131}$, M. Weber ${ }^{115}$, S.G. Weber ${ }^{100}$, D.F. Weiser ${ }^{96}$, J.P. Wessels ${ }^{62}$, U. Westerhoff ${ }^{62}$, A.M. Whitehead ${ }^{92}$, J. Wiechula ${ }^{61}$, J. Wikne ${ }^{21}$, G. Wilk ${ }^{79}$,

J. Wilkinson ${ }^{96}$, G.A. Willems ${ }^{62}$, M.C.S. Williams ${ }^{107}$, B. Windelband ${ }^{96}$, M. Winn ${ }^{96}$, W.E. Witt ${ }^{129}$, S. Yalcin ${ }^{71}$, P. Yang 7 , S. Yano ${ }^{47}$, Z. Yin 7 , H. Yokoyama ${ }^{132,73}$, I.-K. Yoo ${ }^{35,99}$, J.H. Yoon ${ }^{51}$, V. Yurchenko ${ }^{3}$, V. Zaccolo ${ }^{83}$, A. Zaman ${ }^{16}$, C. Zampolli ${ }^{35,107}$, H.J.C. Zanoli ${ }^{123}$, S. Zaporozhets ${ }^{68}$, N. Zardoshti ${ }^{104}$, A. Zarochentsev ${ }^{136}$, P. Závada ${ }^{57}$, N. Zaviyalov ${ }^{102}$, H. Zbroszczyk ${ }^{138}$, M. Zhalov ${ }^{88}$, H. Zhang, 22, X. Zhang 76,7 , Y. Zhang ${ }^{7}$, C. Zhang ${ }^{54}$, Z. Zhang ${ }^{7}$, C. Zhao ${ }^{21}$, N. Zhigareva ${ }^{55}$, D. Zhou ${ }^{7}$, Y. Zhou ${ }^{83}$, Z. Zhou ${ }^{22}$, H. Zhu ${ }^{7,22}$, J. Zhu ${ }^{116,7}$, A. Zichichi' ${ }^{22,27}$,

A. Zimmermann ${ }^{96}$, M.B. Zimmermann ${ }^{62,35}$, G. Zinovjev ${ }^{3}$, J. Zmeskal ${ }^{115}$

\footnotetext{
${ }^{1}$ A.I. Alikhanyan National Science Laboratory (Yerevan Physics Institute) Foundation, Yerevan, Armenia

${ }^{2}$ Benemérita Universidad Autónoma de Puebla, Puebla, Mexico

${ }^{3}$ Bogolyubov Institute for Theoretical Physics, Kiev, Ukraine

${ }^{4}$ Bose Institute, Department of Physics and Centre for Astroparticle Physics and Space Science (CAPSS), Kolkata, India
} 
${ }^{5}$ Budker Institute for Nuclear Physics, Novosibirsk, Russia

${ }^{6}$ California Polytechnic State University, San Luis Obispo, California, USA

${ }^{7}$ Central China Normal University, Wuhan, China

${ }^{8}$ Centre de Calcul de l'IN2P3, Villeurbanne, Lyon, France

${ }^{9}$ Centro de Aplicaciones Tecnológicas y Desarrollo Nuclear (CEADEN), Havana, Cuba

${ }^{10}$ Centro de Investigaciones Energéticas Medioambientales y Tecnológicas (CIEMAT), Madrid, Spain

${ }^{11}$ Centro de Investigación y de Estudios Avanzados (CINVESTAV), Mexico City and Mérida, Mexico

${ }^{12}$ Centro Fermi - Museo Storico della Fisica e Centro Studi e Ricerche "Enrico Fermi', Rome, Italy

${ }^{13}$ Chicago State University, Chicago, Illinois, USA

${ }^{14}$ China Institute of Atomic Energy, Beijing, China

${ }^{15}$ Commissariat à l'Energie Atomique, IRFU, Saclay, France

${ }^{16}$ COMSATS Institute of Information Technology (CIIT), Islamabad, Pakistan

${ }^{17}$ Departamento de Física de Partículas and IGFAE, Universidad de Santiago de Compostela, Santiago de

Compostela, Spain

${ }^{18}$ Department of Physics, Aligarh Muslim University, Aligarh, India

${ }^{19}$ Department of Physics, Ohio State University, Columbus, Ohio, USA

${ }^{20}$ Department of Physics, Sejong University, Seoul, South Korea

${ }^{21}$ Department of Physics, University of Oslo, Oslo, Norway

${ }^{22}$ Department of Physics and Technology, University of Bergen, Bergen, Norway

${ }^{23}$ Dipartimento di Fisica dell'Università 'La Sapienza' and Sezione INFN, Rome, Italy

${ }^{24}$ Dipartimento di Fisica dell'Università and Sezione INFN, Cagliari, Italy

${ }^{25}$ Dipartimento di Fisica dell'Università and Sezione INFN, Trieste, Italy

${ }^{26}$ Dipartimento di Fisica dell'Università and Sezione INFN, Turin, Italy

${ }^{27}$ Dipartimento di Fisica e Astronomia dell'Università and Sezione INFN, Bologna, Italy

${ }^{28}$ Dipartimento di Fisica e Astronomia dell'Università and Sezione INFN, Catania, Italy

${ }^{29}$ Dipartimento di Fisica e Astronomia dell'Università and Sezione INFN, Padova, Italy

${ }^{30}$ Dipartimento di Fisica 'E.R. Caianiello' dell'Università and Gruppo Collegato INFN, Salerno, Italy

${ }^{31}$ Dipartimento DISAT del Politecnico and Sezione INFN, Turin, Italy

${ }^{32}$ Dipartimento di Scienze e Innovazione Tecnologica dell'Università del Piemonte Orientale and INFN Sezione di Torino, Alessandria, Italy

${ }^{33}$ Dipartimento Interateneo di Fisica 'M. Merlin' and Sezione INFN, Bari, Italy

${ }^{34}$ Division of Experimental High Energy Physics, University of Lund, Lund, Sweden

${ }^{35}$ European Organization for Nuclear Research (CERN), Geneva, Switzerland

${ }^{36}$ Excellence Cluster Universe, Technische Universität München, Munich, Germany

${ }^{37}$ Faculty of Engineering, Bergen University College, Bergen, Norway

${ }^{38}$ Faculty of Mathematics, Physics and Informatics, Comenius University, Bratislava, Slovakia

${ }^{39}$ Faculty of Nuclear Sciences and Physical Engineering, Czech Technical University in Prague, Prague, Czech

Republic

${ }^{40}$ Faculty of Science, P.J. Šafárik University, Košice, Slovakia

${ }^{41}$ Faculty of Technology, Buskerud and Vestfold University College, Tonsberg, Norway

${ }^{42}$ Frankfurt Institute for Advanced Studies, Johann Wolfgang Goethe-Universität Frankfurt, Frankfurt, Germany

${ }^{43}$ Gangneung-Wonju National University, Gangneung, South Korea

${ }^{44}$ Gauhati University, Department of Physics, Guwahati, India

${ }^{45}$ Helmholtz-Institut für Strahlen- und Kernphysik, Rheinische Friedrich-Wilhelms-Universität Bonn, Bonn, Germany

${ }^{46}$ Helsinki Institute of Physics (HIP), Helsinki, Finland

${ }^{47}$ Hiroshima University, Hiroshima, Japan

${ }^{48}$ Indian Institute of Technology Bombay (IIT), Mumbai, India

${ }^{49}$ Indian Institute of Technology Indore, Indore, India

${ }^{50}$ Indonesian Institute of Sciences, Jakarta, Indonesia

${ }^{51}$ Inha University, Incheon, South Korea

${ }^{52}$ Institut de Physique Nucléaire d'Orsay (IPNO), Université Paris-Sud, CNRS-IN2P3, Orsay, France

${ }^{53}$ Institute for Nuclear Research, Academy of Sciences, Moscow, Russia

${ }^{54}$ Institute for Subatomic Physics of Utrecht University, Utrecht, Netherlands

${ }^{55}$ Institute for Theoretical and Experimental Physics, Moscow, Russia

${ }^{56}$ Institute of Experimental Physics, Slovak Academy of Sciences, Košice, Slovakia

${ }^{57}$ Institute of Physics, Academy of Sciences of the Czech Republic, Prague, Czech Republic

${ }^{58}$ Institute of Physics, Bhubaneswar, India

${ }^{59}$ Institute of Space Science (ISS), Bucharest, Romania

${ }^{60}$ Institut für Informatik, Johann Wolfgang Goethe-Universität Frankfurt, Frankfurt, Germany 
${ }^{61}$ Institut für Kernphysik, Johann Wolfgang Goethe-Universität Frankfurt, Frankfurt, Germany

${ }^{62}$ Institut für Kernphysik, Westfälische Wilhelms-Universität Münster, Münster, Germany

${ }^{63}$ Instituto de Ciencias Nucleares, Universidad Nacional Autónoma de México, Mexico City, Mexico

${ }^{64}$ Instituto de Física, Universidade Federal do Rio Grande do Sul (UFRGS), Porto Alegre, Brazil

${ }^{65}$ Instituto de Física, Universidad Nacional Autónoma de México, Mexico City, Mexico

${ }^{66}$ Institut Pluridisciplinaire Hubert Curien (IPHC), Université de Strasbourg, CNRS-IN2P3, Strasbourg, France

${ }^{67}$ iThemba LABS, National Research Foundation, Somerset West, South Africa

${ }^{68}$ Joint Institute for Nuclear Research (JINR), Dubna, Russia

${ }^{69}$ Konkuk University, Seoul, South Korea

${ }^{70}$ Korea Institute of Science and Technology Information, Daejeon, South Korea

${ }^{71}$ KTO Karatay University, Konya, Turkey

${ }^{72}$ Laboratoire de Physique Corpusculaire (LPC), Clermont Université, Université Blaise Pascal, CNRS-IN2P3, Clermont-Ferrand, France

${ }^{73}$ Laboratoire de Physique Subatomique et de Cosmologie, Université Grenoble-Alpes, CNRS-IN2P3, Grenoble, France

${ }^{74}$ Laboratori Nazionali di Frascati, INFN, Frascati, Italy

${ }^{75}$ Laboratori Nazionali di Legnaro, INFN, Legnaro, Italy

${ }^{76}$ Lawrence Berkeley National Laboratory, Berkeley, California, USA

${ }^{77}$ Moscow Engineering Physics Institute, Moscow, Russia

${ }^{78}$ Nagasaki Institute of Applied Science, Nagasaki, Japan

${ }^{79}$ National Centre for Nuclear Studies, Warsaw, Poland

${ }^{80}$ National Institute for Physics and Nuclear Engineering, Bucharest, Romania

${ }^{81}$ National Institute of Science Education and Research, Bhubaneswar, India

${ }^{82}$ National Research Centre Kurchatov Institute, Moscow, Russia

${ }^{83}$ Niels Bohr Institute, University of Copenhagen, Copenhagen, Denmark

${ }^{84}$ Nikhef, Nationaal instituut voor subatomaire fysica, Amsterdam, Netherlands

${ }^{85}$ Nuclear Physics Group, STFC Daresbury Laboratory, Daresbury, UK

${ }^{86}$ Nuclear Physics Institute, Academy of Sciences of the Czech Republic, Rež u Prahy, Czech Republic

${ }^{87}$ Oak Ridge National Laboratory, Oak Ridge, Tennessee, USA

${ }^{88}$ Petersburg Nuclear Physics Institute, Gatchina, Russia

${ }^{89}$ Physics Department, Creighton University, Omaha, Nebraska, USA

${ }^{90}$ Physics Department, Panjab University, Chandigarh, India

${ }^{91}$ Physics Department, University of Athens, Athens, Greece

${ }^{92}$ Physics Department, University of Cape Town, Cape Town, South Africa

${ }^{93}$ Physics Department, University of Jammu, Jammu, India

${ }^{94}$ Physics Department, University of Rajasthan, Jaipur, India

${ }^{95}$ Physikalisches Institut, Eberhard Karls Universität Tübingen, Tübingen, Germany

${ }^{96}$ Physikalisches Institut, Ruprecht-Karls-Universität Heidelberg, Heidelberg, Germany

${ }^{97}$ Physik Department, Technische Universität München, Munich, Germany

${ }^{98}$ Purdue University, West Lafayette, Indiana, USA

${ }^{99}$ Pusan National University, Pusan, South Korea

${ }^{100}$ Research Division and ExtreMe Matter Institute EMMI, GSI Helmholtzzentrum für Schwerionenforschung, Darmstadt, Germany

${ }^{101}$ Rudjer Bošković Institute, Zagreb, Croatia

${ }^{102}$ Russian Federal Nuclear Center (VNIIEF), Sarov, Russia

${ }^{103}$ Saha Institute of Nuclear Physics, Kolkata, India

${ }^{104}$ School of Physics and Astronomy, University of Birmingham, Birmingham, UK

${ }^{105}$ Sección Física, Departamento de Ciencias, Pontificia Universidad Católica del Perú, Lima, Peru

${ }^{106}$ Sezione INFN, Bari, Italy

${ }^{107}$ Sezione INFN, Bologna, Italy

${ }^{108}$ Sezione INFN, Cagliari, Italy

${ }^{109}$ Sezione INFN, Catania, Italy

${ }^{110}$ Sezione INFN, Padova, Italy

${ }^{111}$ Sezione INFN, Rome, Italy

${ }^{112}$ Sezione INFN, Trieste, Italy

${ }^{113}$ Sezione INFN, Turin, Italy

${ }^{114}$ SSC IHEP of NRC Kurchatov institute, Protvino, Russia

${ }^{115}$ Stefan Meyer Institut für Subatomare Physik (SMI), Vienna, Austria

${ }^{116}$ SUBATECH, Ecole des Mines de Nantes, Université de Nantes, CNRS-IN2P3, Nantes, France 
${ }^{117}$ Suranaree University of Technology, Nakhon Ratchasima, Thailand

${ }^{118}$ Technical University of Košice, Košice, Slovakia

${ }^{119}$ Technical University of Split FESB, Split, Croatia

${ }^{120}$ The Henryk Niewodniczanski Institute of Nuclear Physics, Polish Academy of Sciences, Cracow, Poland

${ }^{121}$ The University of Texas at Austin, Physics Department, Austin, Texas, USA

${ }^{122}$ Universidad Autónoma de Sinaloa, Culiacán, Mexico

${ }^{123}$ Universidade de São Paulo (USP), São Paulo, Brazil

${ }^{124}$ Universidade Estadual de Campinas (UNICAMP), Campinas, Brazil

${ }^{125}$ Universidade Federal do ABC, Santo Andre, Brazil

${ }^{126}$ University of Houston, Houston, Texas, USA

${ }^{127}$ University of Jyväskylä, Jyväskylä, Finland

${ }^{128}$ University of Liverpool, Liverpool, UK

${ }^{129}$ University of Tennessee, Knoxville, Tennessee, USA

${ }^{130}$ University of the Witwatersrand, Johannesburg, South Africa

${ }^{131}$ University of Tokyo, Tokyo, Japan

${ }^{132}$ University of Tsukuba, Tsukuba, Japan

${ }^{133}$ University of Zagreb, Zagreb, Croatia

${ }^{134}$ Université de Lyon, Université Lyon 1, CNRS/IN2P3, IPN-Lyon, Villeurbanne, Lyon, France

${ }^{135}$ Università di Brescia, Brescia, Italy

${ }^{136}$ V. Fock Institute for Physics, St. Petersburg State University, St. Petersburg, Russia

${ }^{137}$ Variable Energy Cyclotron Centre, Kolkata, India

${ }^{138}$ Warsaw University of Technology, Warsaw, Poland

${ }^{139}$ Wayne State University, Detroit, Michigan, USA

${ }^{140}$ Wigner Research Centre for Physics, Hungarian Academy of Sciences, Budapest, Hungary

${ }^{141}$ Yale University, New Haven, Connecticut, USA

${ }^{142}$ Yonsei University, Seoul, South Korea

${ }^{143}$ Zentrum für Technologietransfer und Telekommunikation (ZTT), Fachhochschule Worms, Worms, Germany

Open Access This is an open access article distributed under the terms of the Creative Commons Attribution License (http://creativecommons.org/licenses/by/4.0), which permits unrestricted use, distribution, and reproduction in any medium, provided the original work is properly cited.

\section{References}

1. ALICE Collaboration (F. Carminati et al.), J. Phys. G 30, 1517 (2004).

2. ALICE Collaboration (B. Alessandro et al.), J. Phys. G 32, 1295 (2006).

3. ALICE Collaboration (B. Abelev et al.), Int. J. Mod. Phys. A 29, 1430044 (2014) arXiv:1402.4476 [nucl-ex].

4. A. Akindinov et al., Nuovo Cimento B 124, 235 (2009).

5. A. Akindinov et al., Eur. Phys. J. Plus 128, 44 (2013).

6. M. Bondila et al., IEEE Trans. Nucl. Sci. 52, 1705 (2005).

7. M. Mota, J. Christiansen, IEEE J. Salid-State Circ. 34, 1360 (1999).

8. ALICE Collaboration (J. Adam et al.), Phys. Rev. C 91, 064905 (2015) arXiv:1412.6828 [nucl-ex].

9. ALICE Collaboration (B. Abelev et al.), Phys. Rev. C 88, 044909 (2013) arXiv:1301.4361 [nucl-ex]. 